\title{
Underlay Spectrum Sharing Techniques with In-band Full-Duplex Systems using Improper Gaussian Signaling
}

\author{
Mohamed Gaafar Student Member, IEEE, Osama Amin, Senior Member, IEEE, Walid Abediseid, Member, IEEE, \\ and Mohamed-Slim Alouini, Fellow, IEEE
}

\begin{abstract}
Sharing the spectrum with in-band full-duplex (FD) primary users (PUs) is a challenging and interesting problem in the underlay cognitive radio (CR) systems. The self-interference introduced at the primary network may dramatically impede the secondary user (SU) opportunity to access the spectrum. To tackle this problem, we use the so-called improper Gaussian signaling. Particularly, we assume the downlink transmission of a SU that uses improper Gaussian signaling while the FD PU pair implements the regular proper Gaussian signaling. First, we derive a closed form expression and an upper bound for the SU and PUs outage probabilities, respectively. Second, we optimize the SU signal parameters to minimize its outage probability while maintaining the required PUs quality-of-service based on the average channel state information (CSI). Moreover, we provide the conditions to reap merits from employing improper Gaussian signaling at the SU. Third, we design the SU signal parameters based on perfect knowledge of its direct link instantaneous CSI and investigate all benefits that can be achieved at both the SU and PUs. Finally, we provide some numerical results that demonstrate the advantages of using improper Gaussian signaling to access the spectrum of the FD PUs.
\end{abstract}

Index Terms-Cognitive radio, underlay, full-duplex, improper Gaussian signaling, interference mitigation, power allocation, spectrum sharing, outage probability, energy efficiency.

\section{INTRODUCTION}

Cognitive radio $(\mathrm{CR})$ is a promising spectrum sharing technology that mitigates the spectrum scarcity, which resulted from the recent tremendous growth of wireless devices and services over the past decade [1]. As many licensed primary users (PUs) block the available spectrum, CR systems exploit the same spectrum resources and allow secondary users (SUs) to coexist with PUs without degrading the PUs quality-of-service (QoS) [2]. CR can operate in three different strategies called, interweave, overlay and underlay spectrum sharing. Underlay CR optimizes the transmitted power in order to be within an acceptable level at the PU receiver side. CR systems can be incorporated with other communication techniques, such as in-band full-duplex (FD) and cooperative communications, to improve the spectrum utilization of the communication networks and the SU performance [3].

FD is a spectral efficient paradigm that allows the communication nodes to transmit and receive simultaneously at the same frequency. FD has recently attracted wide attention

The authors are with Computer, Electrical, and Mathematical Sciences and Engineering (CEMSE) Divison, King Abdullah University of Science and Technology (KAUST), Thuwal, Makkah Province, Saudi Arabia. Email: \{mohamed.gaafar, osama.amin, walid.abediseid, slim.alouini\}@kaust.edu.sa. especially after the progress in self-interference cancellation [3], [4], which gives a great promise in practical realization for next generation wireless networks $[5]^{1}$. In underlay $\mathrm{CR}$ systems, FD is used to compensate the spectral efficiency loss of cooperative systems that are used to increase the SU coverage [6], [7]. In addition to that, FD is used to achieve simultaneous sensing and data transmission for SU, or possibly receive data from the other SU node during the transmission according to the channel conditions [8]. On the other hand, sharing the spectrum of FD PUs is a challenging research problem in underlay cognitive radio that has not addressed before. In this scenario, the PUs are subjected to imperfect self interference cancellation and interference from the SU. Therefore, investigating communication techniques/systems that can relieve the interference signature on the PUs while improving the SU performance becomes imperative.

Improper Gaussian signaling has proven its superiority over proper Gaussian signaling to improve the achievable rate in interference-limited networks [9]-[15]. In CR systems, improper Gaussian signaling is employed in [16]-[19], where the PU is assumed to work in half-duplex mode with proper Gaussian signaling. On the other hand, the SU is assumed to use improper Gaussian signaling and have perfect instantaneous channel state information (CSI) of all PU and SU communication links in [16], [17] and average CSI in [18]. Improper Gaussian signaling showed better performance than proper Gaussian signaling when the PU is not fully loaded. In [19], we considered a challenging spectrum sharing scenario, where the PU pair is assumed to work using in-band FD mode. The SU improper signal parameters are designed to maximize the SU instantaneous rate performance while satisfying PUs minimum fixed rate requirement with the availability of global instantaneous CSI at the SU side.

In this paper ${ }^{2}$, we consider the spectrum sharing problem with in-band FD licensed users, i.e., the PUs. Then, we inspect the possibility of inserting the SU into operation without

\footnotetext{
${ }^{1}$ Self-interference can not be fully canceled due to various reasons such non-linear characteristics of the receiver and imperfect estimation of selfinterference channel. The cancellation procedures includes passive and active techniques based on analog and digital schemes, more details can be found in [3], [4].

${ }^{2}$ This paper extends our work in [20], in which we provide more details and insights for the outage performance analysis and the average CSI based design. We also introduce a design based on the instantaneous SU direct link CSI and investigate its benefits for both SU and PUs. Finally, more numerical results are presented.
} 
deteriorating the PUs QoS based on average or partial CSI. In this regard, we adopt the improper Gaussian signaling for the SU to create a room for spectrum sharing systems. As for measuring the QoS, we use the outage probability for a target rate. To the best of our knowledge, this is the first work that investigates sharing the licensed spectrum of in-band FD PUs based on average or partial CSI. The main contributions of this paper can be summarized as follows:

- We derive a closed form expression for the SU outage probability.

- For the PUs, which are subjected to the SU interference and residual self-interference, we derive a tight upper bound for the outage probability.

- We design the SU signal parameters to minimize its outage probability while maintaining acceptable PUs QoS. In this context, we derive the conditions that provide improvement of adopting the improper Gaussian signaling at the SU over the proper Gaussian signaling.

- Then, we study the benefits of partial CSI knowledge at the SU terminals on the design problem while meeting the lack of coordination assumptions of underlay cognitive radio systems. Specifically, we assume the availability of instantaneous SU direct link and average CSI of other links at the SU terminals. Then, we analyze the performance gain in power saving, outage probability for both SU and PUs, and SU average energy efficiency (EE).

- Finally, with the aid of numerical results, we first examine the accuracy of the PUs derived outage probability bound. Then, we investigate the benefits that can be reaped by employing improper Gaussian signaling for the SU versus different system parameters. And finally, we explore the availability of the SU direct link benefits on power saving, SU and PUs outage probability and SU average EE.

The rest of the paper is organized as follows: In Section II, we introduce the system model of the spectrum sharing system with FD system. In Section III, we derive the outage probability expressions for both SU and PUs. In Section IV, we design the SU signal parameters to minimize the SU outage probability subject to a given QoS for the PUs based on average CSI. In Section V, we study the system performance with perfect CSI knowledge for the SU direct link and look for its advantages on both SU and PU. In Section VI, we present a comprehensive simulation study to illustrate the performance of the proposed improper Gaussian signaling techniques. Finally, Section VI concludes the paper.

Notation: Throughout the rest of the paper, we use |.| to denote the absolute value operation and $[x]^{+}=\max (x, 0)$. $\mathbb{1}_{[x, \infty)}(z)$ is the indicator function where $\mathbb{1}_{[x, \infty)}(z):=1$ if $z \geq x$ and $\mathbb{1}_{[x, \infty)}(z):=0$ for all $z<x . \operatorname{Pr}\{A\}$ denotes the probability of occurrence of an event $\mathrm{A}$. The operator $\mathbb{E}\{$.$\} is$ used to denote the statistical expectation and the mean of a random variable (RV) $X$ is defined as $\bar{X}=\mathbb{E}\{X\}$.

\section{SYSTEM MODEL}

Consider underlay CR system with downlink transmission, where a SU pair coexists with an in-band FD PU pair as depicted in Fig. 1. In this scenario, we have three simultaneous

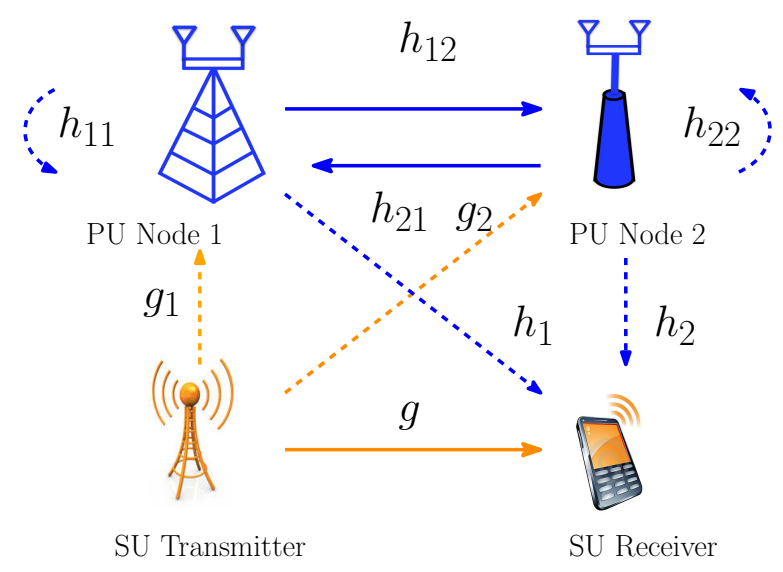

Fig. 1. System model, where the solid and the dash lines are for the desired and the interference signals, respectively.

different transmissions occur in the same network over the same frequency. Before proceeding in describing the system components, we define the following terms:

Definition 1: The variance and pseudo-variance of a zero mean complex random variable $x$ are defined as $\sigma_{x}^{2}=\mathbb{E}\left\{|x|^{2}\right\}$ and $\hat{\sigma}_{x}^{2}=\mathbb{E}\left\{x^{2}\right\}$ respectively [21].

Definition 2: Proper (conventional) Gaussian signaling is a zero mean complex Gaussian signaling scheme whose real and imaginary components are uncorrelated and have equal power. On the other hand, improper Gaussian signaling relaxes the aforementioned assumptions and assumes asymmetric Gaussian components, i.e., correlated and/or with unequal power. Therefore, the proper Gaussian signaling has a zero $\hat{\sigma}_{x}^{2}$, while the improper Gaussian signaling has a non-zero $\hat{\sigma}_{x}^{2}$.

Definition 3: The circularity coefficient $\mathcal{C}_{x}$ measures the degree of impropriety of signal $x$ and is defined as $\mathcal{C}_{x}=$ $\left|\hat{\sigma}_{x}^{2}\right| / \sigma_{x}^{2}$, where $0 \leq \mathcal{C}_{x} \leq 1 . \mathcal{C}_{x}=0$ denotes proper signal and $\mathcal{C}_{x}=1$ denotes maximally improper signal.

The PU nodes are assumed to use zero-mean proper Gaussian signals $x_{i}, i \in\{1,2\}$ with a unit variance, while the SU transmitter uses zero-mean improper Gaussian signals $x_{\mathrm{s}}$ with a unit variance and a circularity coefficient $\mathcal{C}_{x}$. The received signal at the PU node $j$, where $j \in\{1,2\}, i \neq j$, is expressed as

$$
y_{j}=\sqrt{p_{i}} h_{i j} x_{i}+\sqrt{p_{j}} h_{j j} x_{j}+\sqrt{p_{\mathrm{s}}} g_{j} x_{\mathrm{s}}+n_{j},
$$

where $p_{i}$ is the transmitted power of the PU node $i, p_{\mathrm{s}}$ is the SU transmitted power, $n_{j}$ is the noise at the PU node $j$ receiver. As for the communication channels, $h_{i j}$ denotes the channel between PU node $i$ and PU node $j, g_{j}$ represents the interference channel between the SU transmitter and PU node $j$ and $h_{j j}$ represents the residual self interference (RSI) channel of node $j$ after undergoing analog and digital cancellation techniques due to imperfect self-interference cancellation [22]-[25]. It is worth to emphasize that the RSI can not be canceled due to the estimation error of the echo interfering channel [22]. We assume that the RSI is modeled as a zero mean complex Gaussian random variable as in [22]. As for the PUs direct links, we could assume channel reciprocity, i.e., $h_{i j}=h_{j i}$, however it might not be the case when the two PU 
nodes use different spatial antennas locations or the receivers' front end and transmitters' back end are not perfectly matched [26]. Hence, we adopt the general assumption of different forward and reverse PU links.

In the same time, the $\mathrm{SU}$ received signal is expressed as

$$
y_{\mathrm{s}}=\sqrt{p_{\mathrm{s}}} g x_{\mathrm{s}}+\sum_{i=1}^{2} \sqrt{p_{i}} h_{i} x_{i}+n_{\mathrm{s}}
$$

where $n_{\mathrm{S}}$ is the noise at the $\mathrm{SU}$ receiver, $h_{i}$ is the interference channel of PU node $i$ on the SU receiver, $g$ denotes the direct channel between the SU transmitter and receiver. The SU transmitter power has to be adjusted in order not to affect the required QoS of the PUs. The channels in the described system are modeled as slow Rayleigh flat fading channels and the noise at the receivers end is modeled as a white, zero-mean, circularly symmetric, complex Gaussian with variance $\sigma^{2}$. The transmitted signals, channel coefficients and noise components are assumed to be independent of each other except $h_{i j}$ and $h_{j i}$, which may be dependent.

As a result of using improper Gaussian signaling while treating the interference as Gaussian noise at all receivers, the achievable rate for the PU node $i$ is given by [11], [16],

$$
R_{\mathrm{p}_{i}}\left(p_{\mathrm{s}}, \mathcal{C}_{x}\right)=\log _{2}\left(1+\frac{p_{i} \gamma_{\mathrm{p}_{i}}}{p_{j} v_{\mathrm{p}_{j}}+p_{\mathrm{s}} \mathcal{I}_{\mathrm{s}_{j}}+1}\right)+\frac{1}{2} \log _{2}\left(\frac{1-\mathcal{C}_{y_{j}}^{2}}{1-\mathcal{C}_{\mathcal{I}_{j}}^{2}}\right),
$$

where $\gamma_{\mathrm{p}_{i}}=\left|h_{i j}\right|^{2} / \sigma^{2}$ is the channel-to-noise ratio (CNR) of the PU channel from PU node $i$ to PU node $j, \mathcal{I}_{\mathrm{S}_{i}}=$ $\left|g_{i}\right|^{2} / \sigma^{2}$ is the interference CNR of SU to PU node $i$ and $v_{\mathrm{p}_{i}}=\left|h_{i i}\right|^{2} / \sigma^{2}$ is the RSI-CNR of PU node $i, \mathcal{C}_{y_{j}}$ and $\mathcal{C}_{\mathcal{I}_{j}}$ are the circularity coefficients of the received and interferenceplus-noise signals at PU node $j$, respectively, which are given by

$\mathcal{C}_{y_{j}}=\frac{p_{\mathrm{s}} \mathcal{I}_{\mathrm{s}_{j}} \mathcal{C}_{x}}{p_{i} \gamma_{\mathrm{p}_{i}}+p_{j} v_{\mathrm{p}_{j}}+p_{\mathrm{s}} \mathcal{I}_{\mathrm{s}_{j}}+1}, \mathcal{C}_{\mathcal{I}_{j}}=\frac{p_{\mathrm{s}} \mathcal{I}_{\mathrm{s}_{j}} \mathcal{C}_{x}}{p_{j} v_{\mathrm{p}_{j}}+p_{\mathrm{s}} \mathcal{I}_{\mathrm{s}_{j}}+1}$.

It is worth to mention that the additional second term with the " $1 / 2$ " factor in (3) exists as a result of adopting the improper Gaussian signaling, and it vanishes when proper Gaussian signaling is adopted [11]. Furthermore, one can note from (3) and (4) that the second term achieves always rate improvement when improper Gaussian signaling is adopted. After some manipulations, $R_{\mathrm{p}_{i}}\left(p_{\mathrm{s}}, \mathcal{C}_{x}\right)$ can be simplified as

$$
R_{\mathrm{p}_{i}}\left(p_{\mathrm{s}}, \mathcal{C}_{x}\right)=\frac{1}{2} \log _{2}\left(\frac{\left(p_{i} \gamma_{\mathrm{p}_{i}}+p_{j} v_{\mathrm{p}_{j}}+p_{\mathrm{s}} \mathcal{I}_{\mathrm{s}_{j}}+1\right)^{2}-p_{\mathrm{s}}^{2} \mathcal{I}_{\mathrm{s}_{j}}^{2} \mathcal{C}_{x}^{2}}{\left(p_{j} v_{\mathrm{p}_{j}}+p_{\mathrm{s}} \mathcal{I}_{\mathrm{s}_{j}}+1\right)^{2}-p_{\mathrm{s}}^{2} \mathcal{I}_{\mathrm{s}_{j}}^{2} \mathcal{C}_{x}^{2}}\right)
$$

Similarly, the SU achievable rate can be expressed as

$R_{\mathrm{S}}\left(p_{\mathrm{s}}, \mathcal{C}_{x}\right)=\frac{1}{2} \log _{2}\left(\frac{p_{\mathrm{s}}^{2} \gamma_{\mathrm{s}}{ }^{2}\left(1-\mathcal{C}_{x}^{2}\right)}{\left(\sum_{i=1}^{2} p_{i} \mathcal{I}_{\mathrm{p}_{i}}+1\right)^{2}}+\frac{2 p_{\mathrm{s}} \gamma_{\mathrm{s}}}{\sum_{i=1}^{2} p_{i} \mathcal{I}_{\mathrm{p}_{i}}+1}+1\right)$,

where $\gamma_{\mathrm{s}}=|g|^{2} / \sigma^{2}$ is the SU direct CNR between the SU transmitter and receiver and $\mathcal{I}_{\mathrm{p}_{i}}=\left|h_{i}\right|^{2} / \sigma^{2}$ is the PU node $i$ interference CNR to the SU.
The direct, interference and RSI CNR $\gamma_{\mathrm{p}_{i}}, \gamma_{\mathrm{s}}, \mathcal{I}_{\mathrm{p}_{i}}, \mathcal{I}_{\mathrm{s}_{i}}, v_{\mathrm{p}_{i}}$ are exponentially distributed random variables with mean values $\bar{\gamma}_{\mathrm{p}_{i}}, \bar{\gamma}_{\mathrm{s}}, \overline{\mathcal{I}}_{\mathrm{p}_{i}}, \overline{\mathcal{I}}_{\mathrm{s}_{i}}, \bar{v}_{\mathrm{p}_{i}}$, respectively.

One can notice from (5) and (6) that if $\mathcal{C}_{x}=0$, we obtain the well known achievable rates expressions of proper Gaussian signaling. Moreover, if $\mathcal{C}_{x}$ increases, the $\mathrm{SU}$ rate decreases while the PUs rate increases allowing the SU to increase its transmitted power. This merit should be considered carefully to satisfy the PUs QoS requirements and meet the maximum SU power budget.

\section{OUTAGE PROBABILITY ANALYSIS}

Throughout this work, we use the outage probability to measure the performance for both the PUs and SU assuming that both users use fixed rates according to their transmission requirements. For this purpose, we derive expressions of the outage probability for both the PUs and SU in the following subsections.

\section{A. Secondary User Outage Probability}

Assume the SU target rate is $R_{0, \mathrm{~s}}$, then its outage probability is defined as

$$
P_{\text {out }, \mathrm{s}}\left(p_{\mathrm{s}}, \mathcal{C}_{x}\right)=\operatorname{Pr}\left\{R_{\mathrm{s}}\left(p_{\mathrm{s}}, \mathcal{C}_{x}\right)<R_{0, \mathrm{~s}}\right\} .
$$

By substituting (6) in (7), we obtain

$P_{\text {out }, \mathrm{s}}\left(p_{\mathrm{s}}, \mathcal{C}_{x}\right)=\operatorname{Pr}\left\{\frac{p_{\mathrm{s}}^{2} \gamma_{\mathrm{s}}^{2}\left(1-\mathcal{C}_{x}^{2}\right)}{\left(\sum_{i=1}^{2} p_{i} \mathcal{I}_{\mathrm{p}_{i}}+1\right)^{2}}+\frac{2 p_{\mathrm{s}} \gamma_{\mathrm{s}}}{\sum_{i=1}^{2} p_{i} \mathcal{I}_{\mathrm{p}_{i}}+1}-\Gamma_{\mathrm{s}}<0\right\}$,

where $\Gamma_{\mathrm{s}}=2^{2 R_{0, \mathrm{~s}}}-1$. One can show that the conditional SU outage probability (conditioned on $\mathcal{I}_{\mathrm{p}_{1}}$ and $\mathcal{I}_{\mathrm{p}_{2}}$ ) is given by

$$
P_{\text {out }, \mathrm{s}}\left(p_{\mathrm{s}}, \mathcal{C}_{x} \mid \mathcal{I}_{\mathrm{p}_{1}}, \mathcal{I}_{\mathrm{p}_{2}}\right)=1-\exp \left(-\frac{\gamma_{\mathrm{s}}^{\circ}}{\bar{\gamma}_{\mathrm{s}}}\right)
$$

where $\gamma_{\mathrm{s}}^{\circ}$ is the non-negative zero obtained by solving the quadratic inequality in (8) with respect to $\gamma_{s}$, which is found to be

$$
\gamma_{\mathrm{s}}^{\circ}=\frac{\sum_{i=1}^{2} p_{i} \mathcal{I}_{\mathrm{p}_{i}}+1}{\left(1-\mathcal{C}_{x}^{2}\right)} \Psi_{\mathrm{s}}\left(p_{\mathrm{s}}, \mathcal{C}_{x}\right),
$$

where $\Psi_{\mathrm{s}}\left(p_{\mathrm{s}}, \mathcal{C}_{x}\right)=\left(\sqrt{1+\Gamma_{\mathrm{s}}\left(1-\mathcal{C}_{x}^{2}\right)}-1\right) / p_{\mathrm{s}}$. By averaging over the exponential statistics of $\mathcal{I}_{\mathrm{p}_{i}}$, we get

$$
P_{\text {out }, \mathrm{s}}\left(p_{\mathrm{s}}, \mathcal{C}_{x}\right)=1-\frac{\exp \left(-\frac{\Psi_{\mathrm{s}}\left(p_{\mathrm{s}}, \mathcal{C}_{x}\right)}{\bar{\gamma}_{\mathrm{s}}\left(1-\mathcal{C}_{x}^{2}\right)}\right)}{\prod_{j=1}^{2}\left(p_{j} \overline{\mathcal{I}}_{\mathrm{p}_{j}} \frac{\Psi_{\mathrm{s}}\left(p_{\mathrm{s}}, \mathcal{C}_{x}\right)}{\bar{\gamma}_{\mathrm{s}}\left(1-\mathcal{C}_{x}^{2}\right)}+1\right)} .
$$

For the proper signaling case, the above expression reduces to

$$
P_{\text {out }, \mathrm{s}}\left(p_{\mathrm{s}}, 0\right)=1-\frac{\exp \left(-\frac{2^{R_{0, \mathrm{~s}}}-1}{p_{\mathrm{s}} \bar{\gamma}_{\mathrm{s}}}\right)}{\prod_{j=1}^{2}\left(p_{j} \overline{\mathcal{I}}_{\mathrm{p}_{j}} \frac{2^{R_{0, \mathrm{~s}}}-1}{p_{\mathrm{s}} \bar{\gamma}_{\mathrm{s}}}+1\right)} .
$$

while for maximally improper case, the expression reduces to

$$
P_{\text {out }, \mathrm{s}}\left(p_{\mathrm{s}}, 1\right)=\lim _{\mathcal{C}_{x} \rightarrow 1} P_{\text {out }, \mathrm{s}}\left(p_{\mathrm{s}}, \mathcal{C}_{x}\right)=1-\frac{\exp \left(-\Gamma_{\mathrm{s}} / 2 p_{\mathrm{s}} \bar{\gamma}_{\mathrm{s}}\right)}{\prod_{j=1}^{2}\left(\frac{p_{j} \overline{\mathcal{I}}_{\mathrm{p}_{j}} \Gamma_{\mathrm{s}}}{2 p_{\mathrm{s}} \bar{\gamma}_{\mathrm{s}}}+1\right)} .
$$




\section{B. Primary User Outage Probability}

The outage probability of PU node $i$ for a given target rate $R_{0, \mathrm{p}_{i}}$ is defined as

$$
P_{\text {out }, \mathrm{p}_{i}}\left(p_{\mathrm{s}}, \mathcal{C}_{x}\right)=\operatorname{Pr}\left\{R_{\mathrm{p}_{i}}\left(p_{\mathrm{s}}, \mathcal{C}_{x}\right)<R_{0, \mathrm{p}_{i}}\right\}
$$

Substituting (5) in (13), we obtain

$$
\begin{array}{r}
P_{\text {out }, \mathrm{p}_{i}}\left(p_{\mathrm{s}}, \mathcal{C}_{x}\right)=\operatorname{Pr}\left\{\gamma_{\mathrm{p}_{i}}^{2}+\frac{2}{p_{i}}\left(p_{j} v_{\mathrm{p}_{j}}+p_{\mathrm{s}} \mathcal{I}_{\mathrm{s}_{j}}+1\right) \gamma_{\mathrm{p}_{i}}-\right. \\
\left.\frac{\Gamma_{\mathrm{p}_{i}}}{p_{i}^{2}}\left(\left(p_{j} v_{\mathrm{p}_{j}}+p_{\mathrm{s}_{\mathrm{s}}} \mathcal{I}_{\mathrm{s}_{j}}+1\right)^{2}-p_{\mathrm{s}^{2}}^{2} \mathcal{I}_{\mathrm{s}_{j}}^{2} \mathcal{C}_{x}^{2}\right) \leq 0\right\},
\end{array}
$$

where $\Gamma_{\mathrm{p}_{i}}=2^{2 R_{0, \mathrm{p}_{i}}}-1$. Similar to the above subsection, the outage probability of PU node $i$ (conditioned on $\mathcal{I}_{\mathrm{s}_{j}}$ and $v_{\mathrm{p}_{j}}$ ) is given by

$$
P_{\text {out }, \mathrm{p}_{i}}\left(p_{\mathrm{s}}, \mathcal{C}_{x} \mid \mathcal{I}_{\mathrm{s}_{j}}, v_{\mathrm{p}_{j}}\right)=1-\exp \left(-\frac{\gamma_{\mathrm{p}_{i}}^{\circ}}{\bar{\gamma}_{\mathrm{p}_{i}}}\right),
$$

where $\gamma_{\mathrm{p}_{i}}^{\circ}$ represents the non-negative zero obtained by solving the quadratic inequality in (14) with respect to $\gamma_{p_{i}}$, and is found to be

$$
\gamma_{\mathrm{p}_{i}}^{\circ}=\left(p_{j} v_{\mathrm{p}_{j}}+p_{\mathrm{s}} \mathcal{I}_{\mathrm{s}_{j}}+1\right) \Psi_{\mathrm{p}_{i}}\left(\frac{p_{\mathrm{s}} \mathcal{I}_{\mathrm{s}_{j}} \mathcal{C}_{x}}{p_{j} v_{\mathrm{p}_{j}}+p_{\mathrm{s}} \mathcal{I}_{\mathrm{s}_{j}}+1}\right)
$$

where $\Psi_{\mathrm{p}_{i}}(x)=\left(\sqrt{1+\Gamma_{\mathrm{p}_{i}}\left(1-x^{2}\right)}-1\right) / p_{i}$. By averaging over the statistics of $\mathcal{I}_{\mathrm{s}_{j}}$ and $v_{\mathrm{p}_{j}}$ in (15), we obtain

$$
\begin{aligned}
& P_{\text {out }, \mathrm{p}_{i}}\left(p_{\mathrm{s}}, \mathcal{C}_{x}\right)=\mathbb{E}_{\mathcal{I}_{\mathrm{s}_{j}}, v_{\mathrm{p}_{j}}\left\{P_{\text {out }, \mathrm{p}_{i}}\left(p_{\mathrm{s}}, \mathcal{C}_{x} \mid \mathcal{I}_{\mathrm{s}_{j}}, v_{\mathrm{p}_{j}}\right)\right\}} \\
& \quad=1-\int_{0}^{\infty} \int_{0}^{\infty} \frac{\exp \left(-\frac{x}{\overline{\mathcal{I}}_{\mathrm{s}_{j}}}\right) \exp \left(-\frac{y}{\overline{\mathcal{v}}_{\mathrm{p}_{j}}}\right)}{\overline{\mathcal{I}}_{\mathrm{s}_{j}} \bar{v}_{\mathrm{p}_{j}}} \times \\
& \quad \exp \left(-\frac{p_{j} y+p_{\mathrm{s}} x+1}{\bar{\gamma}_{\mathrm{p}_{i}}} \Psi_{\mathrm{p}_{i}}\left(\frac{x p_{\mathrm{s}} \mathcal{C}_{x}}{\left(p_{j} y+p_{\mathrm{s}} x+1\right)}\right)\right) d x d y .
\end{aligned}
$$

Unfortunately, there is no closed form expression for the aforementioned integral except at $\mathcal{C}_{x}=0$, which yields

$$
\begin{aligned}
& P_{\mathrm{out}, \mathrm{p}_{i}}\left(p_{\mathrm{s}}, 0\right)=1- \\
& \left(\frac{p_{i}^{2} \bar{\gamma}_{\mathrm{p}_{i}}^{2} \exp \left(-\frac{2^{R_{0, \mathrm{p}_{i}}-1}}{p_{i} \bar{\gamma}_{\mathrm{p}_{i}}}\right)}{\left(p_{\mathrm{s}} \overline{\mathcal{I}}_{\mathrm{s}_{j}}\left(2^{R_{0, \mathrm{p}_{i}}}-1\right)+p_{i} \bar{\gamma}_{\mathrm{p}_{i}}\right)\left(p_{j} \bar{v}_{\mathrm{p}_{j}}\left(2^{R_{0, \mathrm{p}_{i}}}-1\right)+p_{i} \bar{\gamma}_{\mathrm{p}_{i}}\right)}\right) .
\end{aligned}
$$

For $\mathcal{C}_{x} \neq 0$, we resort to obtaining an upper bound for the outage probability. First, we study the convexity of the exponential term in (15) with respect to $\mathcal{I}_{\mathrm{s}_{j}}$ by defining $F\left(\mathcal{I}_{\mathrm{s}_{j}}\right)=\exp \left(G\left(\mathcal{I}_{\mathrm{s}_{j}}\right)\right)$, where $G\left(\mathcal{I}_{\mathrm{s}_{j}}\right)=$ $\left(D \mathcal{I}_{\mathrm{s}_{j}}+F\right)-\sqrt{A \mathcal{I}_{\mathrm{s}_{j}}^{2}+B \mathcal{I}_{\mathrm{s}_{j}}+C}$ and $A=\frac{p_{\mathrm{s}}^{2}\left(1+\Gamma_{\mathrm{p}_{\mathrm{i}}}\left(1-\mathcal{C}_{x}^{2}\right)\right)}{p_{i}^{2} \bar{\gamma}_{\mathrm{p}_{\mathrm{i}}}^{2}}$, $B=\frac{2 p_{\mathrm{s}}\left(1+\Gamma_{\mathrm{p}_{\mathrm{i}}}\right)\left(1+p_{j} v_{\mathrm{p}_{j}}\right)}{p_{i}^{2} \bar{\gamma}_{\mathrm{p}_{\mathrm{i}}}^{2}}, C=\frac{\left(1+\Gamma_{\mathrm{p}_{\mathrm{i}}}\right)\left(1+p_{j} v_{\mathrm{p}_{j}}\right)^{2}}{p_{i}^{2} \bar{\gamma}_{\mathrm{p}_{\mathrm{i}}}^{2}}, D=\frac{p_{\mathrm{s}}}{p_{i} \bar{\gamma}_{\mathrm{p}_{\mathrm{i}}}}$ and $F=\frac{1+p_{j} v_{\mathrm{p}_{j}}}{p_{i} \bar{\gamma}_{\mathrm{p}_{\mathrm{i}}}}$ are positive constants. The second derivative of $G\left(\mathcal{I}_{\mathrm{s}_{j}}\right)$ is given by

$$
\frac{\partial^{2} G\left(\mathcal{I}_{\mathrm{s}_{j}}\right)}{\partial \mathcal{I}_{\mathrm{s}_{j}}^{2}}=\frac{B^{2}-4 A C}{4\left(C+\mathcal{I}_{\mathrm{s}_{j}}\left(B+A \mathcal{I}_{\mathrm{s}_{j}}\right)\right)^{3 / 2}}>0,
$$

which proves the convexity of $G\left(\mathcal{I}_{\mathrm{s}_{j}}\right)$ and $F\left(\mathcal{I}_{\mathrm{s}_{j}}\right)$ [27]. As a result, we can employ the Jensen's inequality [28] over $\mathcal{I}_{\mathrm{s}_{j}}$ obtaining a conditional upper bound as

$$
\begin{aligned}
P_{\text {out }, \mathrm{p}_{i}}\left(p_{\mathrm{s}}, \mathcal{C}_{x} \mid v_{\mathrm{p}_{j}}\right) \leq & 1-\exp \left(-\frac{p_{j} v_{\mathrm{p}_{j}}+p_{\mathrm{s}} \overline{\mathcal{I}}_{\mathrm{s}_{j}}+1}{\bar{\gamma}_{\mathrm{p}_{i}}} \times\right. \\
& \left.\Psi_{\mathrm{p}_{i}}\left(\frac{p_{\mathrm{s}} \overline{\mathcal{I}}_{\mathrm{s}_{j}} \mathcal{C}_{x}}{p_{j} v_{\mathrm{p}_{j}}+p_{\mathrm{s}} \overline{\mathcal{I}}_{\mathrm{s}_{j}}+1}\right)\right) .
\end{aligned}
$$

Similarly, one can prove that the exponential term in (20) is convex in $v_{\mathrm{p}_{j}}$ and obtain the following upper bound of the PUs outage probability

$$
\begin{array}{r}
P_{\text {out }, \mathrm{p}_{i}}\left(p_{\mathrm{s}}, \mathcal{C}_{x}\right) \leq 1-\exp \left(-\frac{p_{j} \bar{v}_{\mathrm{p}_{j}}+p_{\mathrm{s}} \overline{\mathcal{I}}_{\mathrm{s}_{j}}+1}{\bar{\gamma}_{\mathrm{p}_{i}}} \times\right. \\
\left.\Psi_{\mathrm{p}_{i}}\left(\frac{p_{\mathrm{s}} \overline{\mathcal{I}}_{\mathrm{s}_{j}} \mathcal{C}_{x}}{p_{j} \bar{v}_{\mathrm{p}_{j}}+p_{\mathrm{s}} \overline{\mathcal{I}}_{\mathrm{s}_{j}}+1}\right)\right) \triangleq P_{\text {out }, \mathrm{p}_{i}}^{\mathrm{UB}}\left(p_{\mathrm{s}}, \mathcal{C}_{x}\right) .
\end{array}
$$

Improper Gaussian signaling provides an extra dimension of optimization which is the degree of asymmetry represented in $\mathcal{C}_{x}$. Hence, it provides sort of control of the interference signal dimension, i.e., interference alignment capability [9]. Moreover, at a fixed $p_{\mathrm{s}}$, by increasing $\mathcal{C}_{x}$, there exists a tradeoff as the SU outage probability increases while the PUs outage probability improves, which allow the SU to boost its transmit power. This trade-off can be optimized to enhance the SU outage while preserving the PUs QoS.

\section{Secondary User Transmitted Signal Design}

In this section, we optimize the SU signal parameters to minimize the SU outage probability while maintaining a predetermined PU outage probability for each primary link. We assume the availability of the primary network information the SU side, such as QoS required performance, SIC capability and other system parameters.

First, we state a unified PUs design criterion in order to be satisfied by the SU during the operation with either proper or improper Gaussian signaling. Assume the PU nodes transmit with a fixed rate $R_{0, \mathrm{p}_{i}}$ and a target maximum outage probability threshold of $\mathcal{O}_{\mathrm{p}_{i}}$ while allowing an interference power margin $\mathcal{P}_{\text {int, }} \mathrm{p}_{i}$. Although the licensed band is dedicated locally only to the PU, the interference protection does not only mitigate the RSI. It can also mitigate other interference that can come from other sources such as frequency reuse users in cellular networks [29]. The interference margin is used to ensure certain link budget that is suitable for the transmission QoS. The PUs are assumed to use the maximum power budget $p_{i}$. Thus, the PUs outage probability, from its perspective, is expressed as

$$
\begin{aligned}
\mathcal{O}_{\mathrm{p}_{i}} & =\operatorname{Pr}\left\{\log _{2}\left(1+\frac{p_{i}\left|h_{i j}\right|^{2}}{\sigma^{2}+\mathcal{P}_{\text {int }, \mathrm{p}_{i}}}\right)<R_{0, \mathrm{p}_{i}}\right\} \\
& =1-\exp \left(-\frac{1+\mathcal{I}_{\mathrm{max}, \mathrm{p}_{i}}}{p_{i} \bar{\gamma}_{\mathrm{p}_{i}}}\left(2^{R_{0, \mathrm{p}_{i}}}-1\right)\right),
\end{aligned}
$$

where $\mathcal{I}_{\text {max }, \mathrm{p}_{i}}=\mathcal{P}_{\text {int, } \mathrm{p}_{i}} / \sigma^{2}$ is the maximum allowable margin interference-to-noise ratio (INR) at the receiver of PU node $i$. 
By considering a maximum PUs outage probability threshold $\mathcal{O}_{\mathrm{p}_{i}}, \mathcal{I}_{\max , \mathrm{p}_{i}}$ can be found from (22) as

$$
\mathcal{I}_{\max , \mathrm{p}_{i}}=\left[\frac{\mu_{i}}{\sqrt{1+\Gamma_{\mathrm{p}_{i}}}-1}-1\right]^{+}
$$

where $\mu_{i}=p_{i} \bar{\gamma}_{\mathrm{p}_{i}} \log \left(\frac{1}{1-\mathcal{O}_{p_{i}}}\right)$ and $\log ($.$) is the natural$ logarithm. In the following subsections, we design proper and improper Gaussian signals for the SU to improve its performance, measured by the outage probability, considering a predetermined $\mathcal{O}_{\mathrm{p}_{i}}$ and other system parameters such as $p_{i}$ and $R_{0, \mathrm{p}_{i}}$.

\section{A. Proper Gaussian Signaling Design}

In the case of proper Gaussian signaling, the SU allocates its power in order to minimize its outage probability subject to its own power budget $p_{\mathrm{s}, \max }$ and PUs QoS by solving the following optimization problem,

$$
\begin{array}{cl}
\min _{p_{\mathrm{s}}} & P_{\text {out }, \mathrm{s}}\left(p_{\mathrm{s}}, 0\right) \\
\text { s.t. } & P_{\text {out }, \mathrm{p}_{i}}\left(p_{\mathrm{s}}, 0\right) \leq \mathcal{O}_{p_{i}}, \quad 0<p_{\mathrm{s}} \leq p_{\mathrm{s}, \max } .
\end{array}
$$

The predetermined PUs outage probability constraints in (24) reduce to $p_{\mathrm{s}} \leq p_{\mathrm{s}, \mathrm{p}}^{(i)}$, where $p_{\mathrm{s}, \mathrm{p}}^{(i)}$ is the maximum allowable power that satisfies the PUs required outage probability threshold, which is expressed as

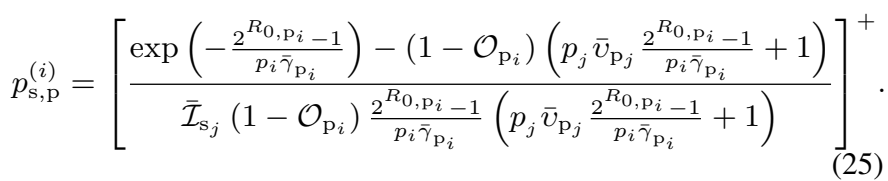

Thus, we can rewrite the optimization problem in (24) as

$$
\begin{array}{ll}
\min _{p_{\mathrm{s}}} & P_{\mathrm{out}, \mathrm{s}}\left(p_{\mathrm{s}}, 0\right) \\
\text { s. t. } & p_{\mathrm{s}} \leq \min \left(p_{\mathrm{s}}^{(1)}, p_{\mathrm{s}}^{(2)}, p_{\mathrm{s}, \max }\right) .
\end{array}
$$

One can prove that $P_{\text {out, } \mathrm{s}}\left(p_{\mathrm{s}}, 0\right)$ is monotonically decreasing in $p_{\mathrm{s}}$, thus the upper bound of the constraint achieves the optimal minimum SU outage probability and is expressed as

$$
p_{\mathrm{s}}=\min \left(p_{\mathrm{s}, \mathrm{p}}^{(1)}, p_{\mathrm{s}, \mathrm{p}}^{(2)}, p_{\mathrm{s}, \max }\right) .
$$

From (25) and (27), the $\mathrm{SU}$ operates if $\exp \left(-\frac{2^{R_{0, \mathrm{p}_{i}}-1}}{p_{i} \bar{\gamma}_{\mathrm{p}_{i}}}\right)>\left(1-\mathcal{O}_{\mathrm{p}_{\rangle}}\right)\left(p_{j} \bar{v}_{\mathrm{p}_{j}} \frac{2^{R_{0, \mathrm{p}_{i}}-1}}{p_{i} \overline{\mathrm{p}}_{\mathrm{p}_{i}}}+1\right)$, which reduces to the following spectrum sharing condition for the maximum allowable margin INR as

$$
\mathcal{I}_{\text {max }, \mathrm{p}_{i}}>\frac{p_{i} \bar{\gamma}_{\mathrm{p}_{i}}}{2^{R_{0, \mathrm{p}_{i}}}-1} \log \left(1+p_{j} \bar{v}_{\mathrm{p}_{j}} \frac{2^{R_{0, \mathrm{p}_{i}}}-1}{p_{i} \bar{\gamma}_{\mathrm{p}_{i}}}\right) .
$$

Otherwise, the SU should remain silent. Therefore, we develop Algorithm I to allocate the optimal power for the SU transmission.

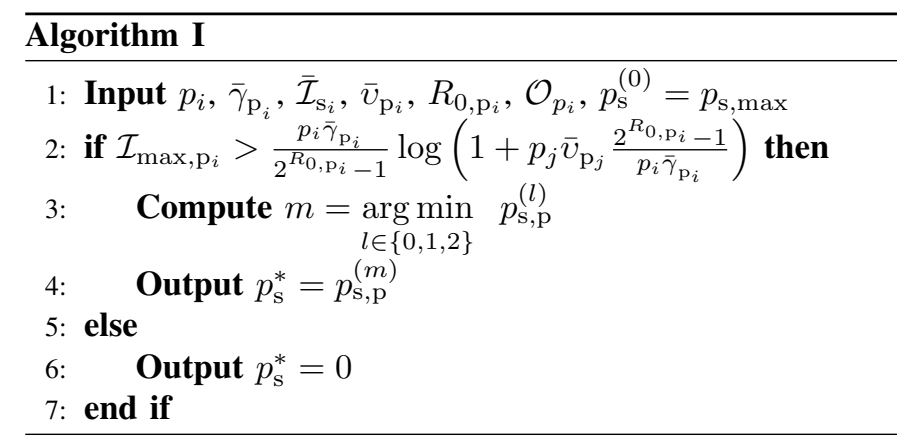

\section{B. Improper Gaussian Signaling Design}

The improper Gaussian signal design aims to tune $p_{\mathrm{s}}$ and $\mathcal{C}_{x}$ to minimize the SU outage while holding a required PUs QoS based on the upper bound derived in (21) achieving the worst case system design. To this end, we formulate the design optimization problem as,

$$
\begin{array}{ll}
\min _{p_{\mathrm{s}}, \mathcal{C}_{x}} & P_{\text {out, } \mathrm{s}}\left(p_{\mathrm{s}}, \mathcal{C}_{x}\right) \\
\text { s. t. } & P_{\text {out }, \mathrm{p}_{i}}^{\mathrm{UB}}\left(p_{\mathrm{s}}, \mathcal{C}_{x}\right) \leq \mathcal{O}_{p_{i}}, 0<p_{\mathrm{s}} \leq p_{\mathrm{s}, \max }, 0 \leq \mathcal{C}_{x} \leq 1 .
\end{array}
$$

Unfortunately, this optimization problem turns to be non-linear and non-convex which makes it hard in general to find its optimal global solution. However, similar to what we did in the proper signaling design, we exploit some monotonicity properties of the objective function and the constraints that lead us to the optimal global solution of (29).

First, based on (21), we express the outage probability constraints in (29) by the following equivalent quadratic inequality in terms of $p_{\mathrm{s}}$ as

$$
\Gamma_{\mathrm{p}_{i}}\left(1-\mathcal{C}_{x}^{2}\right) \overline{\mathcal{I}}_{\mathrm{s}_{j}}^{2} p_{\mathrm{s}}^{2}+2 \Lambda_{i} \overline{\mathcal{I}}_{\mathrm{s}_{j}} p_{\mathrm{s}}-\Upsilon_{i} \leq 0,
$$

where $\Upsilon_{i}=\left(\mu_{i}^{2}+2 \beta_{j} \mu_{i}-\Gamma_{\mathrm{p}_{i}} \beta_{j}^{2}\right), \Lambda_{i}=\left(\beta_{j} \Gamma_{\mathrm{p}_{i}}-\mu_{i}\right), \beta_{j}=$ $\left(p_{j} \overline{\mathrm{p}}_{\mathrm{p}_{j}}+1\right)$. Based on (30), the outage probability constraints are equivalent to $p_{\mathrm{s}} \leq p_{\mathrm{s}}^{(i)}\left(\mathcal{C}_{x}\right)$, where $p_{\mathrm{s}}^{(i)}\left(\mathcal{C}_{x}\right)$ is found by equating the left-hand-side of (30) to zero and then compute the feasible root(s). One can show that if $\Upsilon_{i} \leq 0$, then, $\Lambda_{i}>0$, which results in two negative roots. On the other hand, if $\Upsilon_{i}>$ 0 , then, there is exactly one positive root and one negative root. As a result, the feasible power bound is given by

$$
p_{\mathrm{s}}^{(i)}\left(\mathcal{C}_{x}\right)=\left[\frac{\sqrt{\Lambda_{i}^{2}+\Gamma_{\mathrm{p}_{i}}\left(1-\mathcal{C}_{x}^{2}\right) \Upsilon_{i}}-\Lambda_{i}}{\Gamma_{\mathrm{p}_{i}} \overline{\mathcal{I}}_{\mathrm{s}_{j}}\left(1-\mathcal{C}_{x}^{2}\right)}\right]^{+} .
$$

Thus, the first three constraints in (29) can be equivalently rewritten as

$$
p_{\mathrm{s}}\left(\mathcal{C}_{x}\right) \leq \min \left\{p_{\mathrm{s}}^{(1)}\left(\mathcal{C}_{x}\right), p_{\mathrm{s}}^{(2)}\left(\mathcal{C}_{x}\right), p_{\mathrm{s}, \max }\right\} .
$$

From (31) and (32), the SU is allowed to transmit if $\Upsilon_{i}>0$, which is always valid as long as $\mathcal{I}_{\max , \mathrm{p}_{i}}>p_{j} \bar{v}_{\mathrm{p}_{j}}$. To obtain the distinct intersection points of the aforementioned piecewise function in the interval of interest $0<\mathcal{C}_{x}<1$, first, we can show that $p_{\mathrm{s}}^{(i)}$ is strictly increasing in $\mathcal{C}_{x}$ over the interval (See Appendix A for the proof). Hence, (32) can be described as a piece-wise function with a maximum of four sub-intervals (three breaking points) and a minimum of one interval (zero 
breaking points). The intersection point, $r^{(i)}$, between $p_{\mathrm{s}}^{(i)}$ and $p_{\mathrm{s}, \max }$ is found from

$$
r^{(i)}=\sqrt{1+\frac{2\left(p_{\mathrm{s}, \max } \overline{\mathcal{I}}_{\mathrm{s}_{j}}\right) \Lambda_{i}-\Upsilon_{i}}{\Gamma_{\mathrm{p}_{i}}\left(p_{\mathrm{s}, \max } \overline{\mathcal{I}}_{\mathrm{s}_{j}}\right)^{2}}},
$$

which exists if $p_{s}^{(i)}(0)<p_{\mathrm{s}, \max }$ and $p_{\mathrm{s}}^{(i)}(1)>p_{\mathrm{s}, \max }$. Furthermore, the intersection between $p_{\mathrm{s}}^{(1)}$ and $p_{\mathrm{s}}^{(2)}$ in the interval of interest, if they are not identical, is $r^{(3)}=\sqrt{(1-\kappa)}$, where $\kappa$ is computed from

$\kappa=\frac{4 \Gamma_{\mathrm{p}_{1}} \Gamma_{\mathrm{p}_{2}} \overline{\mathcal{I}}_{\mathrm{s}_{1}} \overline{\mathcal{I}}_{\mathrm{s}_{2}}\left(\Gamma_{\mathrm{p}_{1}} \Lambda_{2} \overline{\mathcal{I}}_{\mathrm{s}_{2}}-\Gamma_{\mathrm{p}_{2}} \Lambda_{1} \overline{\mathcal{I}}_{\mathrm{s}_{1}}\right)\left(\Lambda_{2} \Upsilon_{1} \overline{\mathcal{I}}_{\mathrm{s}_{1}}-\Lambda_{1} \Upsilon_{2} \overline{\mathcal{I}}_{\mathrm{s}_{2}}\right)}{\left(\Gamma_{\mathrm{p}_{2}} \Upsilon_{1} \overline{\mathcal{I}}_{\mathrm{s}_{1}}^{2}-\Gamma_{\mathrm{p}_{1}} \Upsilon_{2} \overline{\mathcal{I}}_{\mathrm{s}_{2}}^{2}\right)^{2}}$

which exists if $p_{s}^{(i)}(0)<p_{s}^{(j)}(0)$ and $p_{s}^{(i)}(1)>p_{s}^{(j)}(1)$. The identical case i.e., $p_{\mathrm{s}}^{(1)}=p_{\mathrm{s}}^{(2)}$ occurs if the links' received CNR at the receiver of PU node $i$ are equal. In such scenario, we have at most two sub-intervals (one breaking point) that can be obtained from (33) if exists.

Define the sub-interval boundaries points as $\mathcal{C}_{x}^{(z)}$, where $z$ is an integer number in $[1, k+1], k$ is the number of distinct intersection points, i.e. $k \in\{0,1,2,3\}, \mathcal{C}_{x}^{(0)}=0, \mathcal{C}_{x}^{(k+1)}=1$ and $\mathcal{C}_{x}^{(1)}, \mathcal{C}_{x}^{(2)}$ and $\mathcal{C}_{x}^{(3)}$ are the ordered distinct intersection points (if exist).

Thereafter, we divide the optimization problem in (29) into $(k+1)$ sub-problems, where each sub-problem is defined in a specific range $\mathcal{C}_{x}^{(z-1)} \leq \mathcal{C}_{x} \leq \mathcal{C}_{x}^{(z)}$. We can show that $P_{\text {out }, \mathrm{s}}\left(p_{\mathrm{s}}, \mathcal{C}_{x}\right)$ is monotonically decreasing in $p_{\mathrm{s}}$ for a fixed $\mathcal{C}_{x}$. Hence, $p_{\mathrm{s}}$ is assigned the upper bound of (32) to minimize the outage probability. Then, we check the minimum of the three functions in (32) in each sub-interval and substitute the value of $p_{\mathrm{s}}$ in $P_{\text {out }, \mathrm{s}}\left(p_{\mathrm{s}}, \mathcal{C}_{x}\right)$ obtaining $k+1$ sub-problems, where the $z^{\text {th }}$ sub-problem is written as

$$
\begin{aligned}
\mathfrak{P}_{z}: \min _{\mathcal{C}_{x}} P_{\text {out }, \mathrm{s}}\left(\mathcal{C}_{x}\right) \\
\text { s. t. } \mathcal{C}_{x}^{(z-1)}<\mathcal{C}_{x} \leq \mathcal{C}_{x}^{(z)} .
\end{aligned}
$$

To solve the $\mathfrak{P}_{z}$ problem, we have two cases, either $p_{\mathrm{s}}=$ $p_{\mathrm{s}, \max }$ or $p_{\mathrm{s}}=p_{\mathrm{s}}^{(m)}\left(\mathcal{C}_{x}\right)$, where $m$ denotes the index of the minimum of the two functions $p_{\mathrm{s}}^{(i)}$ in the sub-interval. Firstly, if $p_{\mathrm{s}}=p_{\mathrm{s}}^{(m)}$ in (32), $P_{\mathrm{out}, \mathrm{s}}\left(\mathcal{C}_{x}\right)$ reduces to

$$
P_{\text {out }, \mathrm{s}}\left(\mathcal{C}_{x}\right)=1-\frac{\mathcal{Y}^{2} \mathcal{G}_{m}^{2}\left(\bar{\gamma}_{\mathrm{s}}\right) \exp \left(-\frac{1}{\mathcal{Y} \mathcal{G}_{m}\left(\bar{\gamma}_{\mathrm{s}}\right)}\right)}{\prod_{j=1}^{2}\left(p_{j} \overline{\mathcal{I}}_{\mathrm{p}_{j}}+\mathcal{Y} \mathcal{G}_{m}\left(\bar{\gamma}_{\mathrm{s}}\right)\right)},
$$

where $\mathcal{Y}=\sqrt{1-\mathcal{C}_{x}^{2}} /\left(\sqrt{1+\left(1-\mathcal{C}_{x}^{2}\right) \Gamma_{\mathrm{s}}}-1\right)$ and $\mathcal{G}_{m}(z)=p_{\mathrm{s}}^{(m)} z \sqrt{1-\mathcal{C}_{x}^{2}}$. Thus, $P_{\text {out }, \mathrm{s}}$ in (36) is monotonically decreasing in $\mathcal{C}_{x}$ if (See Appendix B for the proof.)

$$
R_{0, \mathrm{~s}} \leq \log _{2}\left(\frac{\mu_{m} \sqrt{1+\Gamma_{\mathrm{p}_{\mathrm{m}}}}}{\beta_{j} \Gamma_{\mathrm{p}_{\mathrm{m}}}-\mu_{m}}\right), \quad j \neq m .
$$

Therefore, the optimal solution pair in this case is $\left(p_{\mathrm{o}}^{(z)}, \mathcal{C}_{\mathrm{o}}^{(z)}\right)=\left(p_{s}^{(m)}\left(\mathcal{C}_{x}^{(z)}\right), \mathcal{C}_{x}^{(z)}\right)$. Otherwise, it is monotonically increasing and hence, $\left(p_{\mathrm{o}}^{(z)}, \mathcal{C}_{\mathrm{o}}^{(z)}\right)=$
$\left(p_{s}^{(m)}\left(\mathcal{C}_{x}^{(z-1)}\right), \mathcal{C}_{x}^{(z-1)}\right)$.

Remark: Given that the maximum INR for PU node $i$ exceeds $p_{j} \bar{v}_{\mathrm{p}_{j}}$, the inequality in (37) gives the condition for improper Gaussian signals to be beneficial for the SU transmission.

Secondly, if $p_{\mathrm{s}}=p_{\mathrm{s}, \max }$ in (32), then by substituting its value in the SU outage probability, we obtain $P_{\text {out }, \mathrm{s}}\left(\mathcal{C}_{x}\right)=P_{\text {out }, \mathrm{s}}\left(p_{\mathrm{s}, \max }, \mathcal{C}_{x}\right)$ that is monotonically increasing in $\mathcal{C}_{x}$ and can be easily shown in a similar way to the proof in Appendix B. Hence, the optimal solution pair is $\left(p_{\mathrm{o}}^{(z)}, \mathcal{C}_{\mathrm{o}}^{(z)}\right)=\left(p_{\mathrm{s}, \max }, \mathcal{C}_{x}^{(z-1)}\right)$. At the end, we pick the global optimal pair $\left(p_{\mathrm{o}}^{(z)}, \mathcal{C}_{\mathrm{o}}^{(z)}\right)$ that minimizes the objective function $P_{\text {out, } \mathrm{s}}\left(p_{\mathrm{s}}, \mathcal{C}_{x}\right)$. Based on the aforementioned analysis, we develop Algorithms II to find the distinct intersection points and the optimal solution pairs in $z$ regions, then find the pair, $\left(p_{\mathrm{S}}^{*}, \mathcal{C}_{x}^{*}\right)$, with minimum SU outage probability from

$$
\left(p_{\mathrm{s}}^{*}, \mathcal{C}_{x}^{*}\right)=\arg \min p_{\text {out }, \mathrm{s}}\left(p_{\mathrm{o}}^{(z)}, \mathcal{C}_{\mathrm{o}}^{(z)}\right) .
$$

The proposed algorithms are non-iterative and they have

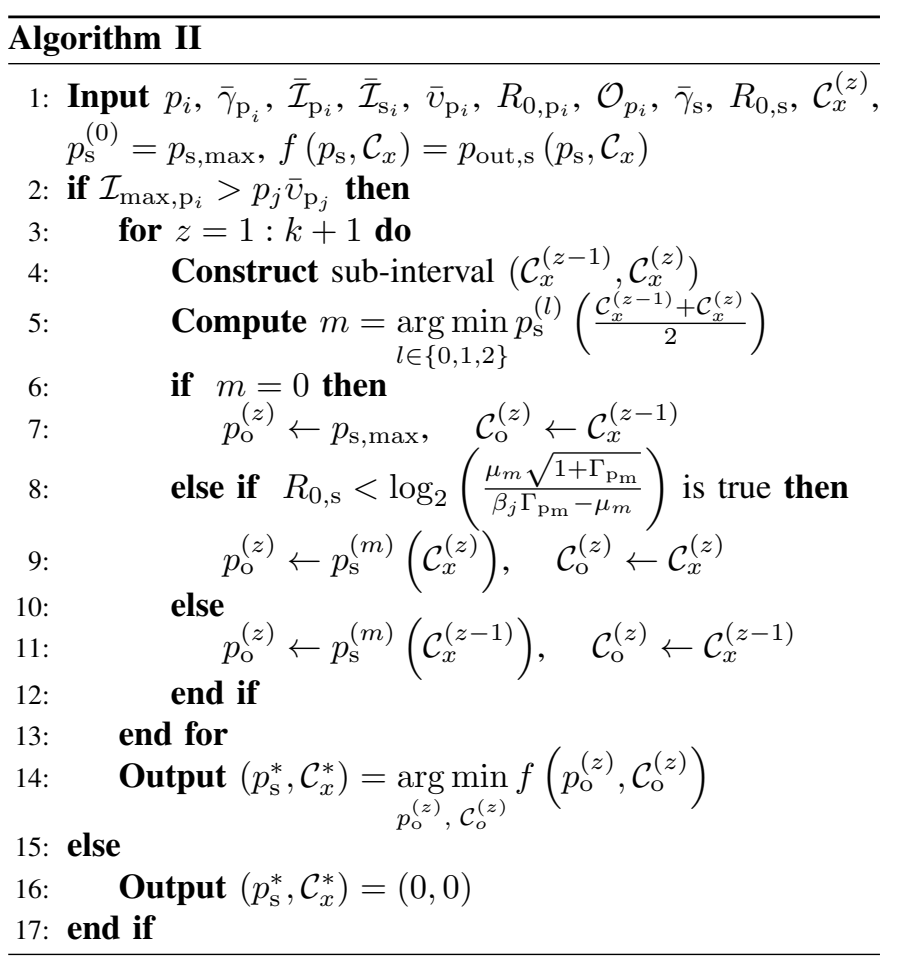

a fixed complexity where they perform fixed number of comparisons to evaluate the optimal solution.

\section{Performance Analysis with Secondary user DIRECT LINK CSI}

In this section, we analyze the performance of the underlay spectrum sharing system using improper Gaussian signaling with the availability of the SU direct link instantaneous CSI. Such an assumption meets the lack of coordination feature between the SU and the PUs in underlay CR systems, where the PUs are unaware of the underlay cognitive radio operation. First, we present analysis of the the SU outage probability with the knowledge of its direct link CSI. Then, we design the SU improper Gaussian signal based on the CSI knowledge 
to improve the SU outage probability performance. After that, we investigate the benefits of the instantaneous direct link CSI (IDL-CSI) based design versus the average CSI (A-CSI) based design on both SU and PUs.

\section{A. Secondary User Outage Probability with Direct Link CSI}

In this subsection, we derive the SU outage probability with perfect SU direct link $\mathrm{CSI}^{3}$. First, We can rewrite (8) as

$$
\begin{array}{r}
P_{\text {out }, \mathrm{s}}^{\mathrm{DL}}\left(p_{\mathrm{s}}, \mathcal{C}_{x}\right)=\operatorname{Pr}\left\{\Gamma_{\mathrm{s}}(\Xi+1)^{2}-2 p_{\mathrm{s}} \gamma_{\mathrm{s}}(\Xi+1)-\right. \\
\left.p_{\mathrm{s}}^{2} \gamma_{\mathrm{s}}^{2}\left(1-\mathcal{C}_{x}^{2}\right) \geq 0\right\},
\end{array}
$$

where $\Xi=\sum_{i=1}^{2} p_{i} \mathcal{I}_{\mathrm{p}_{i}}$. By solving the quadratic inequality in (39) with respect to $\Xi$ and after some manipulations, we obtain

$$
P_{\text {out }, \mathrm{s}}^{\mathrm{DL}}\left(p_{\mathrm{s}}, \mathcal{C}_{x}\right)=\operatorname{Pr}\left\{\Xi \geq \zeta\left(p_{\mathrm{s}}, \mathcal{C}_{x}\right)\right\}=\int_{\zeta\left(p_{\mathrm{s}}, \mathcal{C}_{x}\right)}^{\infty} f_{\Xi}(z) d z
$$

where $\zeta\left(p_{\mathrm{s}}, \mathcal{C}_{x}\right)$ is defined as

$$
\zeta\left(p_{\mathrm{s}}, \mathcal{C}_{x}\right)=\frac{\gamma_{\mathrm{s}}\left(1-\mathcal{C}_{x}^{2}\right)}{\Psi_{\mathrm{s}}\left(p_{\mathrm{s}}, \mathcal{C}_{x}\right)}-1
$$

and $f_{\Xi}(z)$ is the probability density function (PDF) of the random variable $\Xi$. Since $p_{1} \mathcal{I}_{\mathrm{p}_{1}}$ and $p_{2} \mathcal{I}_{\mathrm{p}_{2}}$ are independent exponential random variables with mean $p_{1} \overline{\mathcal{I}}_{\mathrm{p}_{1}}$ and $p_{2} \overline{\mathcal{I}}_{\mathrm{p}_{2}}$, respectively, then $\Xi$ is a hypoexponential random variable with two rate parameters $1 / p_{1} \overline{\mathcal{I}}_{\mathrm{p}_{1}}$ and $1 / p_{2} \overline{\mathcal{I}}_{\mathrm{p}_{2}}$. Hence, according to [30], its PDF, assuming that $p_{1} \overline{\mathcal{I}}_{\mathrm{p}_{1}} \neq p_{2} \overline{\mathcal{I}}_{\mathrm{p}_{2}}$, is found to be

$$
f_{\Xi}\left(z \mid p_{1} \overline{\mathcal{I}}_{\mathrm{p}_{1}} \neq p_{2} \overline{\mathcal{I}}_{\mathrm{p}_{2}}\right)=\sum_{\substack{i=1 \\ j \neq i}}^{2} \frac{\exp \left(-\frac{z}{p_{i} \overline{\mathcal{I}}_{\mathrm{p}_{i}}}\right)}{p_{i} \overline{\mathcal{I}}_{\mathrm{p}_{i}}-p_{j} \overline{\mathcal{I}}_{\mathrm{p}_{j}}} \mathbb{1}_{[0, \infty)}(z) .
$$

For $p_{1} \overline{\mathcal{I}}_{\mathrm{p}_{1}}=p_{2} \overline{\mathcal{I}}_{\mathrm{p}_{2}}$, the PDF of $\Xi$ reduces to

$$
f_{\Xi}\left(z \mid p_{1} \overline{\mathcal{I}}_{\mathrm{p}_{1}}=p_{2} \overline{\mathcal{I}}_{\mathrm{p}_{2}}\right)=\frac{z \exp \left(-\frac{z}{p_{1} \overline{\mathcal{I}}_{\mathrm{p}_{1}}}\right)}{\left(p_{1} \overline{\mathcal{I}}_{\mathrm{p}_{1}}\right)^{2}} \mathbb{1}_{[0, \infty)}(z),
$$

which represents the Erlang distribution with parameters, shape $k=2$ and rate $\lambda=1 / p_{1} \overline{\mathcal{I}}_{\mathrm{p}_{1}}$ [31].

From (40), we note that $P_{\mathrm{out}, \mathrm{s}}^{\mathrm{DL}}\left(p_{\mathrm{s}}, \mathcal{C}_{x}\right)$ attains its maximum value of unity if $\zeta\left(p_{\mathrm{s}}, \mathcal{C}_{x}\right) \leq 0$. Thus, the $\mathrm{SU}$ outage probability in this scenario can be rewritten as

$$
P_{\text {out }, \mathrm{s}}^{\mathrm{DL}}\left(p_{\mathrm{s}}, \mathcal{C}_{x}\right)=\left\{\begin{array}{cc}
1, & \gamma_{\mathrm{s}} \leq \frac{\Psi_{\mathrm{s}}\left(p_{\mathrm{s}}, \mathcal{C}_{x}\right)}{1-\mathcal{C}_{x}^{2}} \\
P_{\mathrm{out}, \mathrm{s}}^{\mathrm{DL}-\mathrm{T}}\left(p_{\mathrm{s}}, \mathcal{C}_{x}\right), & \gamma_{\mathrm{s}}>\frac{\Psi_{\mathrm{s}}\left(p_{\mathrm{s}}, \mathcal{C}_{x}\right)}{1-\mathcal{C}_{x}^{2}}
\end{array},\right.
$$

where we obtain $100 \%$ outage of the SU transmission for highly faded SU direct link channel regardless of the PUs interference level on the SU, thus no-transmission should be adopted at the SU side. On the other hand, when the SU

\footnotetext{
${ }^{3}$ Perfect CSI assumption is used to provide a performance limit of the proposed system.
}

has a good direct link channel, it transmits. The transmission condition is defined according to (44)

$$
\gamma_{\mathrm{s}}>\frac{\Psi_{\mathrm{s}}\left(p_{\mathrm{s}}, \mathcal{C}_{x}\right)}{1-\mathcal{C}_{x}^{2}}
$$

Then, we obtain the corresponding outage probability under perfect knowledge of $\gamma_{\mathrm{s}}$ as

$$
\left\{\begin{array}{cl}
P_{\text {out }, \mathrm{s}}^{\mathrm{DL}-\mathrm{T}}\left(p_{\mathrm{s}}, \mathcal{C}_{x}\right)= & p_{1} \overline{\mathcal{I}}_{\mathrm{p}_{1}} \neq p_{2} \overline{\mathcal{I}}_{\mathrm{p}_{2}} \frac{p_{i} \overline{\mathcal{I}}_{\mathrm{p}_{i}} \exp \left(-\frac{\zeta\left(p_{\mathrm{s}}, \mathcal{C}_{x}\right)}{p_{i} \overline{\mathcal{I}}_{\mathrm{p}_{i}}}\right)}{p_{i} \mathcal{I}_{\mathrm{P}_{i}}-p_{j} \mathcal{I}_{\mathrm{p}_{j}}}, \\
\left(1+\frac{\zeta\left(p_{\mathrm{s}}, \mathcal{C}_{x}\right)}{p_{1} \overline{\mathcal{I}}_{\mathrm{p}_{1}}}\right) \exp \left(-\frac{\zeta\left(p_{\mathrm{s}}, \mathcal{C}_{x}\right)}{p_{1} \mathcal{I}_{\mathrm{p}_{1}}}\right), & p_{1} \overline{\mathcal{I}}_{\mathrm{p}_{1}}=p_{2} \overline{\mathcal{I}}_{\mathrm{p}_{2}}
\end{array} .\right.
$$

According to the availability of SU CSI, the SU will not be allowed to access the spectrum if (45) is not valid. On the other hand, if the transmission condition is valid, i.e., (45), then SU outage is governed not only by $\gamma_{\mathrm{s}}$, but also by the PU interference links on the SU.

For the proper case, i.e., $\mathcal{C}_{x}=0$, the $\mathrm{SU}$ outage probability, when transmitting, reduces to

$$
\begin{aligned}
& P_{\mathrm{out}, \mathrm{s}}^{\mathrm{DL}-\mathrm{T}}\left(p_{\mathrm{s}}, 0\right)= \\
& \left\{\begin{array}{cc}
\sum_{\substack{i=1 \\
j \neq i}}^{2} \frac{p_{i} \overline{\mathcal{I}}_{\mathrm{p}_{i}} \exp \left(-\frac{\frac{p_{\mathrm{s}} \gamma_{\mathrm{s}}}{R_{0, \mathrm{~s}}-1}-1}{p_{i} \mathcal{I}_{\mathrm{p}_{i}}}\right)}{p_{i} \overline{\mathcal{I}}_{\mathrm{p}_{i}}-p_{j} \overline{\mathcal{I}}_{\mathrm{p}_{j}}}, & p_{1} \overline{\mathcal{I}}_{\mathrm{p}_{1}} \neq p_{2} \overline{\mathcal{I}}_{\mathrm{p}_{2}} \\
\left(1+\frac{\frac{p_{\mathrm{s}} \gamma_{\mathrm{s}}}{2^{R_{0}, \mathrm{~s}}-1}}{p_{1} \overline{\mathcal{I}}_{\mathrm{p}_{1}}}\right) \exp \left(-\frac{\frac{p_{\mathrm{s}} \gamma_{\mathrm{s}}}{2^{R_{0, \mathrm{~s}}}-1}}{p_{1} \mathcal{\mathcal { I }}_{\mathrm{p}_{1}}}\right), & p_{1} \overline{\mathcal{I}}_{\mathrm{p}_{1}}=p_{2} \overline{\mathcal{I}}_{\mathrm{p}_{2}}
\end{array} .\right.
\end{aligned}
$$

while for maximally improper case, i.e., $\mathcal{C}_{x}=1$, it yields

$$
\begin{aligned}
& \lim _{\mathcal{C}_{x} \rightarrow 1} P_{\text {out }, \mathrm{s}}^{\mathrm{DL}-\mathrm{T}}\left(p_{\mathrm{s}}, \mathcal{C}_{x}\right)=
\end{aligned}
$$

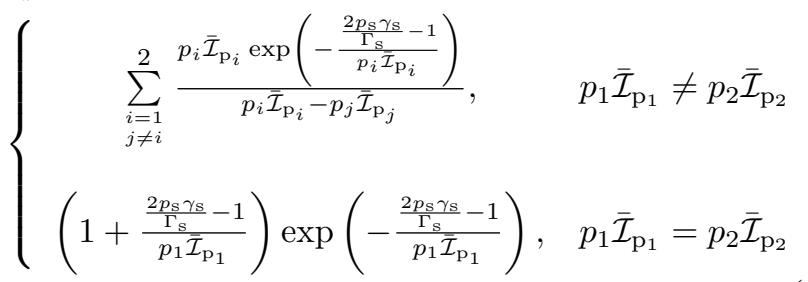

\section{B. Design of Secondary User Signal Parameters Based on Perfect Direct Link CSI}

In this subsection, we aim to design the SU signal parameters, i.e., $p_{\mathrm{s}}$ and $\mathcal{C}_{x}$, in the improper Gaussian signaling case, and merely $p_{\mathrm{s}}$, in the proper case, in order to satisfy the PUs QoS and the boundary values for these parameters. Therefore, we solve the optimization problem for the proper proper case

$$
\begin{array}{cl}
\min _{p_{\mathrm{s}}} & P_{\mathrm{out}, \mathrm{s}}^{\mathrm{DL}}\left(p_{\mathrm{s}}, 0\right) \\
\text { s.t. } & P_{\text {out }, \mathrm{p}_{i}}\left(p_{\mathrm{s}}, 0\right) \leq \mathcal{O}_{p_{i}}, \quad 0<p_{\mathrm{s}} \leq p_{\mathrm{s}, \max },
\end{array}
$$

and the following optimization problem for the improper case,

$$
\begin{array}{ll}
\min _{p_{\mathrm{s}}, \mathcal{C}_{x}} & P_{\text {out,s }}^{\mathrm{DL}}\left(p_{\mathrm{s}}, \mathcal{C}_{x}\right) \\
\text { s. t. } & P_{\text {out }, \mathrm{p}_{i}}^{\mathrm{UB}}\left(p_{\mathrm{s}}, \mathcal{C}_{x}\right) \leq \mathcal{O}_{p_{i}}, 0 \leq p_{\mathrm{s}} \leq p_{\mathrm{s}, \max }, 0 \leq \mathcal{C}_{x} \leq 1 .
\end{array}
$$


First, for the proper Gaussian signaling case, one can show that $P_{\text {out, } \mathrm{s}}^{\mathrm{DL}}\left(p_{\mathrm{s}}, 0\right)$ is monotonically decreasing in $p_{\mathrm{s}}$. Thus the optimal minimum SU outage probability is achieved by allocating the SU power to the minimum of the three functions in (27) as long as the transmission condition is valid, i.e., (45). Otherwise the SU should stay silent because a unity SU outage probability is expected to be achieved. Therefore, as a modification of the previous section, the SU needs to check the validity of transmission condition in (45) at $\mathcal{C}_{x}=0$, to stay silent or to transmit with a power that is computed from Algorithm I.

For the improper case, if $p_{\mathrm{s}}=p_{\mathrm{s}}^{(m)}, m=\{1,2\}$ in (32), then the SU outage probability in the transmission scenario, i.e., (45) is valid, can be written in terms of $\mathcal{C}_{x}$ as

$$
\begin{aligned}
& P_{\text {out }, \mathrm{s}}^{\mathrm{DL}-\mathrm{T}}\left(\mathcal{C}_{x}\right)= \\
& \left\{\begin{array}{cc}
\sum_{\substack{i=1 \\
j \neq i}}^{2} \mathcal{K}_{i, j} \exp \left(-\frac{\mathcal{Y} \mathcal{G}_{m}\left(\gamma_{\mathrm{s}}\right)-1}{p_{i} \overline{\mathcal{I}}_{\mathrm{p}_{i}}}\right), & p_{1} \overline{\mathcal{I}}_{\mathrm{p}_{1}} \neq p_{2} \overline{\mathcal{I}}_{\mathrm{p}_{2}} \\
\left(1+\frac{\mathcal{Y} \mathcal{G}_{m}\left(\gamma_{\mathrm{s}}\right)-1}{p_{1} \overline{\mathcal{I}}_{\mathrm{p}_{1}}}\right) \exp \left(-\frac{\mathcal{Y} \mathcal{G}_{m}\left(\gamma_{\mathrm{s}}\right)-1}{p_{1} \overline{\mathcal{I}}_{\mathrm{p}_{1}}}\right), & p_{1} \overline{\mathcal{I}}_{\mathrm{p}_{1}}=p_{2} \overline{\mathcal{I}}_{\mathrm{p}_{2}}
\end{array}\right.
\end{aligned}
$$

where $\mathcal{K}_{i, j}=p_{i} \overline{\mathcal{I}}_{\mathrm{p}_{i}} /\left(p_{i} \overline{\mathcal{I}}_{\mathrm{p}_{i}}-p_{j} \overline{\mathcal{I}}_{\mathrm{p}_{j}}\right)$. Thus, $P_{\text {out }, \mathrm{s}}\left(\mathcal{C}_{x}\right)$ is monotonically decreasing in $\mathcal{C}_{x}$, when the condition in (37) is true (See Appendix $\mathrm{C}$ for the proof). Moreover, if $p_{\mathrm{s}}=p_{\mathrm{s}, \max }$ in (32), $P_{\text {out,s }}\left(\mathcal{C}_{x}\right)$ is monotonically increasing in $\mathcal{C}_{x}$ which can be proven similar to the proof in Appendix $C$. Thus, same steps are applied to obtain the optimal $\left(p_{\mathrm{O}}^{(z)}, \mathcal{C}_{\mathrm{O}}^{(z)}\right)$ pairs in each of the sub-intervals defined by the boundary points $\mathcal{C}_{x}^{(z)}$, i.e., steps 6 to 12 in Algorithm II. At the end, we calculate the global optimal pair $\left(p_{\mathrm{s}}^{*}, \mathcal{C}_{x}^{*}\right)$ from

$$
\left(p_{\mathrm{s}}^{*}, \mathcal{C}_{x}^{*}\right)=\arg \min p_{\text {out }, \mathrm{s}}^{\mathrm{DL}}\left(p_{\mathrm{o}}^{(z)}, \mathcal{C}_{\mathrm{o}}^{(z)}\right),
$$

which can be further simplified and formulated as (See Appendix $\mathrm{D}$ for the proof):

$$
\left(p_{\mathrm{s}}^{*}, \mathcal{C}_{x}^{*}\right)=\arg \min \frac{1-\left(\mathcal{C}_{o}^{(z)}\right)^{2}}{\Psi_{\mathrm{s}}\left(p_{o}^{(z)}, \mathcal{C}_{o}^{(z)}\right)} .
$$

It is clear from (53) that the optimal parameters are independent of $\gamma_{\mathrm{s}}$. Thus, the availability of the direct link CSI information determines only whether the SU can transmit or not as can be seen from (44) and (45). As a result, by knowing the direct link CSI, the SU is able to save some of its transmit power and hence boost its average EE as will be discussed in the following subsections.

\section{Benefits of SU Direct Link CSI}

In this subsection, we investigate the benefits obtained by the SU through perfect CSI knowledge of the SU direct link.

1) $S U$ power saving: In the improper design of the spectrum sharing system based on the average statistics of the channel coefficients in Section IV, it is clear that the SU always transmits if the maximum INR for user $i$ exceeds $p_{j} \bar{v}_{\mathrm{p}_{j}}$. On the contrary, based on perfect CSI of the SU direct link, the SU stays silent if the transmission condition (45) is not satisfied because it yields a unity SU outage probability. Thus, the probability of the power saving event is expressed as

$$
\begin{aligned}
P_{\text {saving }} & =\operatorname{Pr}\left\{\gamma_{\mathrm{s}} \leq \frac{\Psi_{\mathrm{s}}\left(p_{\mathrm{s}}, \mathcal{C}_{x}\right)}{\left(1-\mathcal{C}_{x}^{2}\right)}\right\} \\
& =1-\exp \left(-\frac{\Psi_{\mathrm{s}}\left(p_{\mathrm{s}}, \mathcal{C}_{x}\right)}{\bar{\gamma}_{\mathrm{s}}\left(1-\mathcal{C}_{x}^{2}\right)}\right),
\end{aligned}
$$

which represents also the portion of time that the SU does not consume energy and and stays idle.

2) Improving the SU average energy efficiency: The improper Gaussian signaling design tends to use more power to improve the SU design and relieve its impact on the PUs by increasing the circularity coefficient. This can be concluded from the monotonically decreasing characteristics of the $P_{\text {out, }}$ in $\mathcal{C}_{x}$ discussed in Sections (IV-B and V-B). Since the SU CSI offers some power saving advantages, then the average EE of the SU system is expected to improve in this scenario. In the following, we analyze the average EE of the SU system when using the SU direct link CSI comparing to the design case based on the average statistics of CSI.

The EE of the SU system is defined as the ratio between the data that is successfully delivered to the receiver and the corresponding total energy consumption [32]. Firstly, for the average statistics based design, the SU always transmits and the average $\mathrm{EE}$ of the $\mathrm{SU}$ is expressed as

$$
\bar{\eta}_{\mathrm{EE}}^{\mathrm{Avg}}=\frac{R_{0, \mathrm{~s}}\left(1-p_{\text {out }, \mathrm{s}}\left(p_{\mathrm{s}}, \mathcal{C}_{x}\right)\right)}{\kappa p_{\mathrm{s}}+p_{c}},
$$

where $\kappa$ is the reciprocal power amplifier efficiency and $p_{\mathrm{c}}$ is the circuits power consumption.

Secondly, for the case of known direct link CSI, the average $\mathrm{EE}$ is defined when the SU is allowed to transmit, i.e., $\gamma_{\mathrm{s}} \geq$ $\Psi_{\mathrm{s}}\left(p_{\mathrm{s}}, \mathcal{C}_{x}\right) /\left(1-\mathcal{C}_{x}^{2}\right)$, as

$$
\bar{\eta}_{\mathrm{EE}}^{\mathrm{DL}}=\mathbb{E}_{\gamma_{\mathrm{s}}}\left\{\eta_{\mathrm{EE}}\left(\gamma_{\mathrm{s}} \mid \gamma_{\mathrm{s}}>\frac{\Psi_{\mathrm{s}}\left(p_{\mathrm{s}}, \mathcal{C}_{x}\right)}{1-\mathcal{C}_{x}^{2}}\right)\right\},
$$

where $\eta_{\mathrm{EE}}$ represents the $\mathrm{EE}$ of the $\mathrm{SU}$ at a specific direct link CSI, which is defined as

$$
\eta_{\mathrm{EE}}\left(\gamma_{\mathrm{s}}\right)=\frac{R_{0, \mathrm{~s}}\left(1-p_{\text {out }, \mathrm{s}}^{\mathrm{DL}}\left(p_{\mathrm{s}}, \mathcal{C}_{x}\right)\right)}{P_{\mathrm{T}}\left(\gamma_{\mathrm{s}}\right)}
$$

where $P_{\mathrm{T}}\left(\gamma_{\mathrm{s}}\right)$ is the total power used to deliver the data and defined as

$$
P_{\mathrm{T}}\left(\gamma_{\mathrm{s}}\right)=\left(\kappa p_{\mathrm{s}}+p_{\mathrm{c}}\right) \mathbb{1}_{\left[\frac{\Psi_{\mathrm{s}}\left(p_{\mathrm{s}}, \mathcal{C}_{x}\right)}{\left(1-\mathcal{C}_{x}^{2}\right)}, \infty\right)}\left(\gamma_{\mathrm{s}}\right) .
$$

Here, we have assumed that the SU transmitter consumes negligible power in the idle/sleep mode. From (56), the SU average EE can be written as

$$
\bar{\eta}_{\mathrm{EE}}^{\mathrm{DL}}=\frac{\int_{\Psi_{\mathrm{s}}\left(p_{\mathrm{s}}, \mathcal{C}_{x}\right)}^{1-\mathcal{C}_{x}^{2}} \eta_{\mathrm{EE}}\left(\gamma_{\mathrm{s}}\right) \exp \left(-\frac{x}{\bar{\gamma}_{\mathrm{s}}}\right) d x}{\bar{\gamma}_{\mathrm{s}}\left(1-P_{\text {saving }}\right)} .
$$

After evaluating the integral in (59), we obtain

$$
\bar{\eta}_{\mathrm{EE}}^{\mathrm{DL}}=\frac{R_{0, \mathrm{~s}}}{\left(\kappa p_{\mathrm{s}}+p_{c}\right) \prod_{j=1}^{2}\left(p_{j} \overline{\mathcal{I}}_{\mathrm{p}_{j}} \frac{\Psi_{\mathrm{s}}\left(p_{\mathrm{s}}, \mathcal{C}_{x}\right)}{\bar{\gamma}_{\mathrm{s}}\left(1-\mathcal{C}_{x}^{2}\right)}+1\right)} .
$$


From (55) and (60), we compute the improvement in the average $\mathrm{EE}, \mathcal{E}$, after simplification as

$$
\mathcal{E}=\frac{\bar{\eta}_{\mathrm{EE}}^{\mathrm{DL}}}{\bar{\eta}_{\mathrm{EE}}^{\mathrm{Avg}}}=\frac{1}{1-P_{\text {saving }}}
$$

3) PUs Outage Probability Enhancement: As discussed in the previous 2 points, the SU can make use of the CSI of direct link in order to save its transmit power and enhance the average EE performance. Moreover, when the SU detects such outage events and decides to abandon its transmission, the interference impact on the PUs will be significantly alleviated and hence, the outage performance of the PUs will be improved as follows

$$
\begin{aligned}
& P_{\text {out, } \mathrm{p}_{i}}^{\mathrm{DL}}\left(p_{\mathrm{s}}, \mathcal{C}_{x}\right)= \\
& P_{\text {saving }} P_{\text {out }, \mathrm{p}_{i}}(0,0)+\left(1-P_{\text {saving }}\right) P_{\text {out }, \mathrm{p}_{i}}\left(p_{\mathrm{s}}, \mathcal{C}_{x}\right) \\
& =P_{\text {out }, \mathrm{p}_{i}}\left(p_{\mathrm{s}}, \mathcal{C}_{x}\right)-P_{\text {saving }} \underbrace{\left(P_{\text {out }, \mathrm{p}_{i}}\left(p_{\mathrm{s}}, \mathcal{C}_{x}\right)-P_{\text {out }, \mathrm{p}_{i}}(0,0)\right)}_{(a)} \\
& \leq P_{\text {out }, \mathrm{p}_{i}}\left(p_{\mathrm{s}}, \mathcal{C}_{x}\right),
\end{aligned}
$$

where $(a)$ follows from the fact that interference free system performance is better than systems subjected to any type of interference. Thus, SU direct link channel knowledge provides a protection for the PUs performance. This merit may be used by other cognitive users in the same system to access the spectrum without deteriorating the PUs QoS.

\section{NumeriCAl Results}

In this section, we present some numerical examples and simulations that validate the introduced analysis and investigate the benefits of employing improper Gaussian signaling in spectrum sharing with FD PUs. First, we compare the proposed PUs outage probability upper bound with the exact expression that is computed numerically. Thereafter, we exploit these bounds to design the SU signal parameters. The design is based on minimizing the SU outage probability while maintaining certain QoS requirements for the PUs based on the average CSI. We also examine the effect of RSI channel of the employment of improper Gaussian signaling at the SU. Moreover, we investigate the proposed system design in case of the perfect knowledge of the direct link CSI and verify the benefits that the SU can attain in terms of power saving and hence average EE improvement. Furthermore, we provide numerical simulations of the benefit that is achieved at the PUs side.

Throughout this Section, we use the following general system parameters for all examples, unless otherwise specified. For the PU nodes, we assume $R_{0, \mathrm{p}_{i}}=0.5 \mathrm{~b} / \mathrm{s} / \mathrm{Hz}$ with a maximum power budget $p_{i}=1 \mathrm{~W}$. The communications channels are characterized as, $\bar{\gamma}_{\mathrm{p}_{i}}=25 \mathrm{~dB}, \overline{\mathcal{I}}_{\mathrm{p}_{i}}=3 \mathrm{~dB}$, $\bar{v}_{\mathrm{p}_{i}}=5 \mathrm{~dB}$. We assume that the required PUs outage probability threshold $\mathcal{O}_{p_{i}}=0.01$. The $\mathrm{SU}$ is assumed to target $R_{0, \mathrm{~s}}=0.5 \mathrm{~b} / \mathrm{s} / \mathrm{Hz}$ using $p_{\mathrm{s}, \max }=1 \mathrm{~W}$. The SU channels' parameters are assumed to be $\overline{\mathcal{I}}_{\mathrm{s}_{i}}=13 \mathrm{~dB}$ and $\bar{\gamma}_{\mathrm{s}}=20 \mathrm{~dB}$. For the IDL-CSI based design, we used $10^{6}$ independent channel realizations for the direct link of the SU in the simulation.

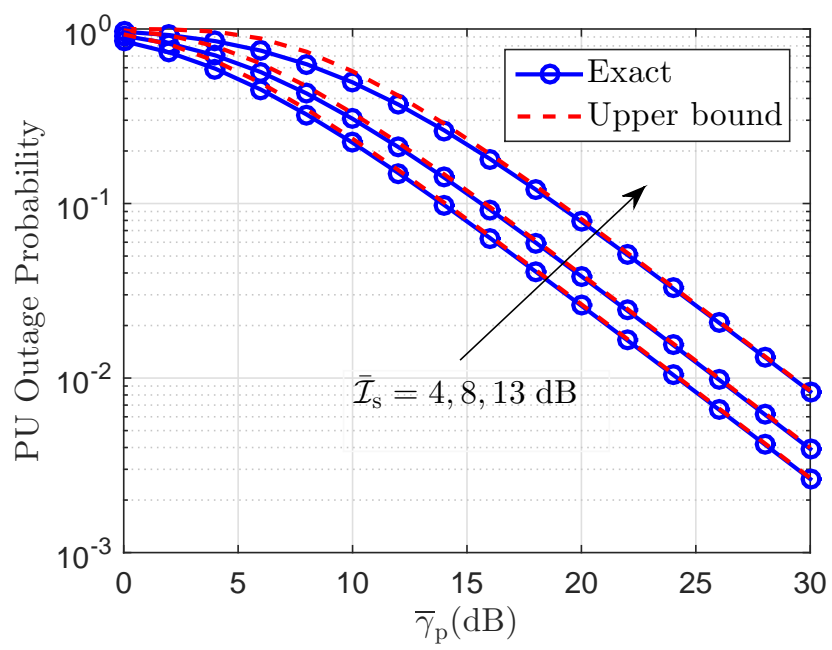

Fig. 2. A comparison between the exact PUs outage probability and the upper bound versus $\bar{\gamma}_{\mathrm{p}}$ for $\overline{\mathcal{I}}_{\mathrm{s}}=4,8,13 \mathrm{~dB}$.

Example 1: This example compares the upper bound computed from (21) with the exact value computed by evaluating the expectations in (17) numerically. We assume $\bar{\gamma}_{\mathrm{p}_{i}}=\bar{\gamma}_{\mathrm{p}}$, $\mathcal{C}_{x}=0.5$ and $\overline{\mathcal{I}}_{\mathrm{s}_{i}}=\overline{\mathcal{I}}_{\mathrm{s}}=4,8,13 \mathrm{~dB}$. As shown in Fig. 2 , the upper bound is tight to the exact outage probability for different $\overline{\mathcal{I}}_{\mathrm{s}}$. Similar results are observed for different $R_{0, \mathrm{p}_{i}}$.

Example 2: In this example, we inspect the benefits of designing the improper Gaussian signaling for SU over the conventional proper Gaussian signaling design. We assume the pair $\left(\overline{\mathcal{I}}_{\mathrm{s}_{1}}, \overline{\mathcal{I}}_{\mathrm{s}_{2}}\right)$ has has the following values, $(0,4) \mathrm{dB},(4,8)$ $\mathrm{dB}$ and $(13,13) \mathrm{dB}$. The proper design is based on Algorithm I, while the improper design is based on Algorithm II. Fig. 3 shows the SU outage probability versus $\bar{\gamma}_{\mathrm{s}}$ for different pairs of $\overline{\mathcal{I}}_{\mathrm{s}_{1}}$. For $\left(\overline{\mathcal{I}}_{\mathrm{s}_{1}}, \overline{\mathcal{I}}_{\mathrm{s}_{2}}\right)=(0,4) \mathrm{dB}$, there is no gain from using improper signaling. In this case, the interference channel is weak, which allow the SU with proper signaling to improve its performance (minimize its outage probability) by increasing the transmitted power and employing the maximum budget. As we observed from the improper design, $p_{\mathrm{s}}$ tends to increase with $\mathcal{C}_{x}$ as can be seen in (31), but since $p_{\mathrm{s}}(0) \simeq p_{\mathrm{s}, \max }$, then the improper solution reduces approximately to the proper design. As the SU interference channels $\overline{\mathcal{I}}_{\mathrm{s}_{i}}$ become stronger, proper signaling system uses less power to meet PUs QoS requirement while improper signaling can use more power to improve its outage probability performance while compensating for its interference impact on the PUs by increasing the circularity coefficient. Fig. 3 shows a $1.5-3.5 \mathrm{~dB}$ improvement resulting from adopting improper Gaussian signaling.

As for the corresponding PUs outage performance, Table I lists the exact outage probabilities for proper and improper Gaussian signaling schemes. At low interference values, i.e. the first pair in Table I, the impact of the SU on the PUs outage probability is tolerable where the improper Gaussian signaling solution reduces to proper one using the the maximum power budget. As $\mathcal{I}_{\mathrm{s}_{i}}$ increases, the power allocation of the proper signaling design is based on the worst scenario, i.e., stronger 


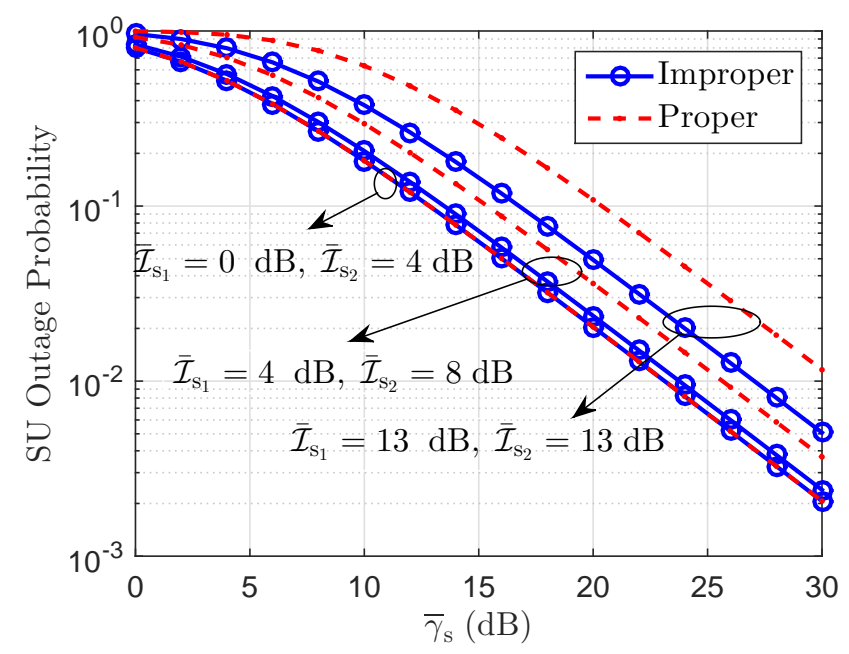

Fig. 3. SU outage probability for proper and improper Gaussian signaling versus $\bar{\gamma}_{\mathrm{s}}$ for $\left(\overline{\mathcal{I}}_{\mathrm{s}_{1}}, \overline{\mathcal{I}}_{\mathrm{s}_{2}}\right)=(0,4),(4,8),(13,13) \mathrm{dB}$ pairs.

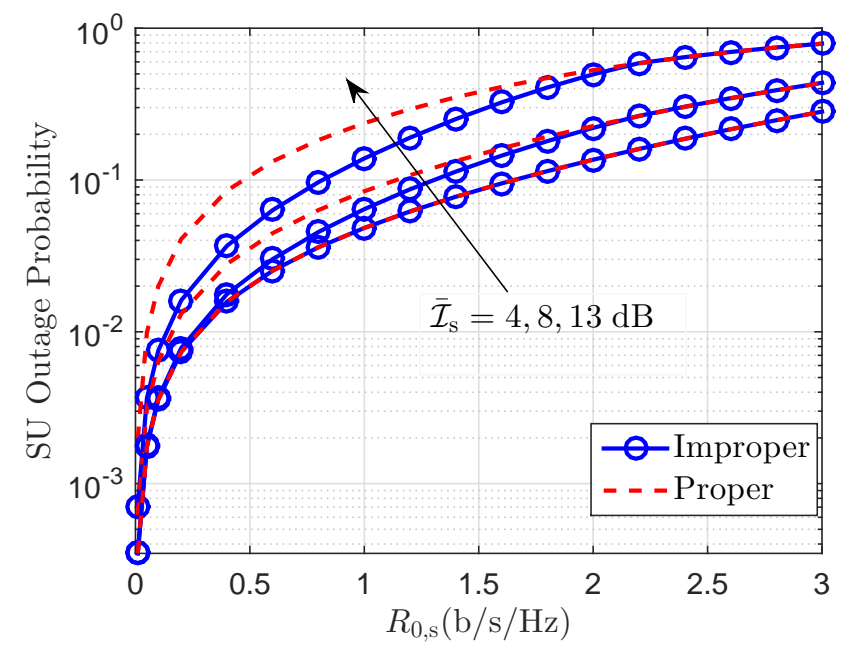

Fig. 4. SU outage probability for proper and improper Gaussian signaling versus $R_{0, \mathrm{~s}}$ for $\overline{\mathcal{I}}_{\mathrm{s}}=4,8,13 \mathrm{~dB}$.

SU link to the PUs, meeting the PUs outage constraint, i.e., 0.01. On the other hand, the improper design allows using more transmit power that reaches the total budget by relieving the interference impact by increasing the circularity coefficient.

Example 3: Fig. 4 plots the SU outage probability versus different SU target rates $R_{0, \mathrm{~s}}$. We assume that $\overline{\mathcal{I}}_{\mathrm{s}_{i}}=\overline{\mathcal{I}}_{\mathrm{s}}=4,8,13$ dB. Similar to the previous example, it is clear that improper Gaussian signaling system achieves superior performance that the proper one when the SU interference channel to the PUs is strong. However, at high SU target rates, there is no gain from employing improper signaling as can be deduced from the condition in (37).

Example 4: This example investigates the impact of RSICNR in limiting the CR operation and compares between its effect on both proper and improper Gaussian signaling based systems. We assume $\bar{v}_{\mathrm{p}_{i}}=\bar{v}_{\mathrm{p}}$. For this purpose, we plot the SU outage probability versus $\bar{v}_{\mathrm{p}}$ for different values of

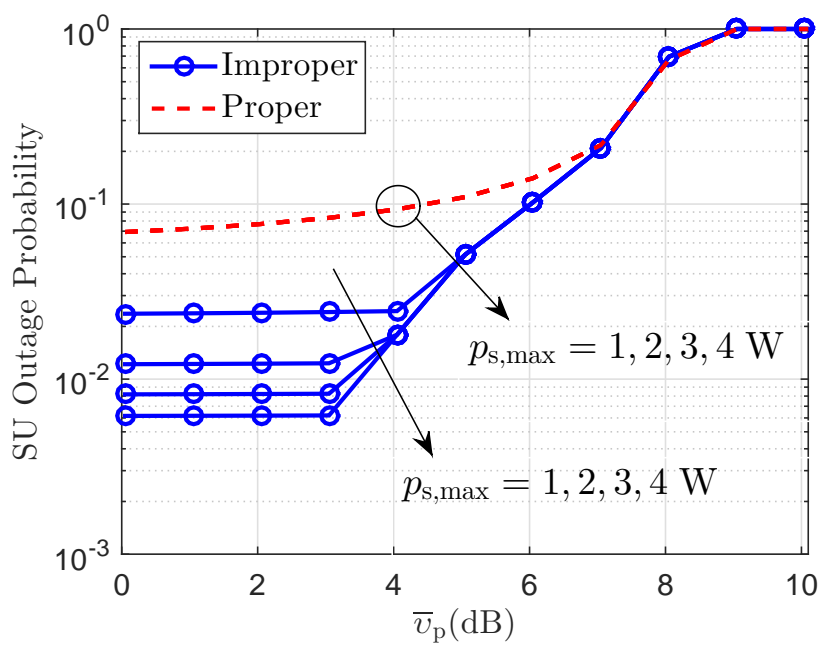

Fig. 5. SU outage probability for proper and improper Gaussian signaling versus $\bar{v}_{\mathrm{p}}$ for $p_{\mathrm{s}, \max }=1,2,3,4 \mathrm{~W}$.

$p_{\mathrm{s}, \max }$ in Fig. 5. We observe that improper Gaussian signaling achieves better performance than the proper Gaussian signaling system at low values of $\bar{v}_{\mathrm{p}}$. Although the proper Gaussian signaling system cannot get benefits from increasing the power budget, the improper Gaussian signaling tends to use the total budget efficiently. It relieves the interference effect on PUs by increasing $\mathcal{C}_{x}$, which compensates for the interference impact as can be seen in (3). On the other hand, at high RSI-CNR values, both proper and improper fail to operate properly.

Example 5: In this example, we compare the design of the proper/improper Gaussian signaling design based on both ACSI and IDL-CSI. To this end, we plot SU outage probability versus $\bar{\gamma}_{\mathrm{s}}$ for different $\overline{\mathcal{I}}_{\mathrm{s}_{i}}$ in Fig. 6 . The simulation result shows a perfect match between the A-CSI and IDL-CSI based designs for both the proper and improper Gaussian signaling schemes. For low $\bar{\gamma}_{\mathrm{s}}$, the SU outage occurs mainly because of its direct link and the IDL-CSI based design saves the SU power and stay silent. As a result, no data is delivered to the SU receiver and outage is reported. On the other hand, for large $\bar{\gamma}_{\mathrm{s}}$, the SU outage is mainly controlled by the PUs interference link and the optimal signal parameters of IDLCSI based system does not depend on $\overline{\gamma_{\mathrm{s}}}$ as can seen from (53).

Example 6: This example studies first the benefit of knowledge of $\gamma_{\mathrm{s}}$ represented in the power saving. Fig. 7 shows the SU power saving percentage versus $\bar{\gamma}_{\mathrm{s}}$ for different SU target rates of $R_{0, \mathrm{~s}}$. It can be seen that at lower values of $\bar{\gamma}_{\mathrm{s}}$, the SU can save more power instead of transmitting and an outage event occurs. At higher values of $\bar{\gamma}_{\mathrm{s}}$, the SU channel conditions are good enough to achieve its target rate. Moreover, if the SU target rate increases, the probability of having an outage event increases and hence, the SU preferably stays idle and saves its transmit power.

Example 7: To investigate the average EE improvement benefit as a result of perfect knowledge of $\gamma_{\mathrm{s}}$, Fig. 8 plots the SU average EE for the SU improper Gaussian signaling design based A-CSI and IDL-CSI using simulations and 
TABLE I

Exact PUs outage probabilities for proper and improper Gaussian signaling for different pairs of $\mathcal{I}_{\mathrm{s}_{i}}$

\begin{tabular}{|c|l|c|c|c|}
\multicolumn{2}{c|}{} & $\overline{\mathcal{I}}_{\mathrm{s}_{1}}=0 \mathrm{~dB}$ & $\overline{\mathcal{I}}_{\mathrm{s}_{1}}=4 \mathrm{~dB}$ & $\overline{\mathcal{I}}_{\mathrm{s}_{1}}=13 \mathrm{~dB}$ \\
\hline \multirow{2}{*}{ Proper } & $\overline{\mathcal{I}}_{\mathrm{s}_{2}}=4 \mathrm{~dB}$ & $\overline{\mathcal{I}}_{\mathrm{s}_{2}}=8 \mathrm{~dB}$ & $\overline{\mathcal{I}}_{\mathrm{s}_{2}}=13 \mathrm{~dB}$ \\
\cline { 2 - 5 } & $P_{\text {out }, \mathrm{p}_{1}}$ & 0.0087 & 0.0100 & 0.0100 \\
\hline \multirow{2}{*}{ Improper } & $P_{\text {out, } \mathrm{p}_{1}}$ & 0.0067 & 0.0073 & 0.0100 \\
\cline { 2 - 5 } & $P_{\text {out, } \mathrm{p}_{2}}$ & 0.0087 & 0.0092 & 0.0088 \\
\hline
\end{tabular}

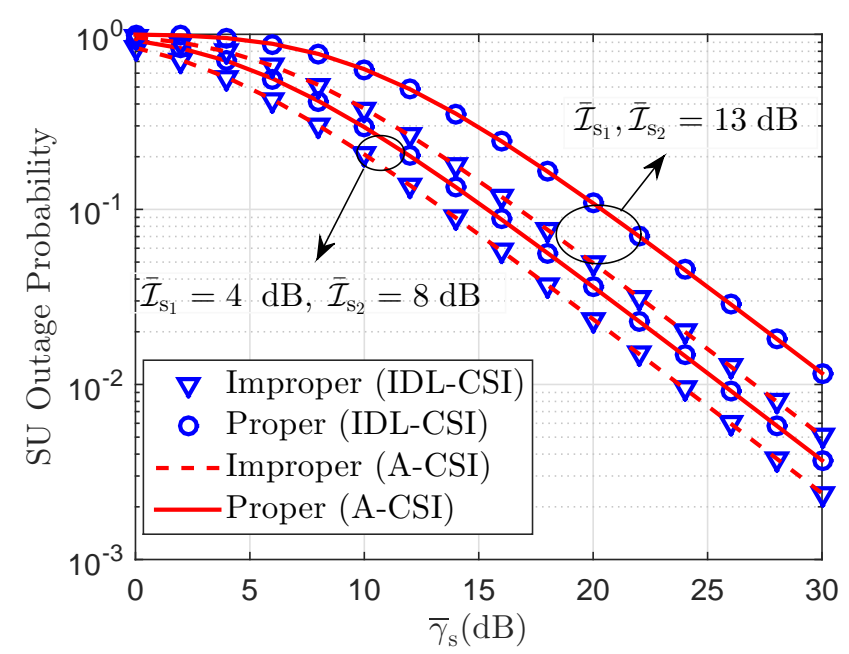

Fig. 6. SU outage probability for A-CSI and IDL-CSI based designs versus $\bar{\gamma}_{\mathrm{s}}$ for $\left(\overline{\mathcal{I}}_{\mathrm{s}_{1}}, \overline{\mathcal{I}}_{\mathrm{s}_{2}}\right)=(4,8),(13,13) \mathrm{dB}$ pairs.

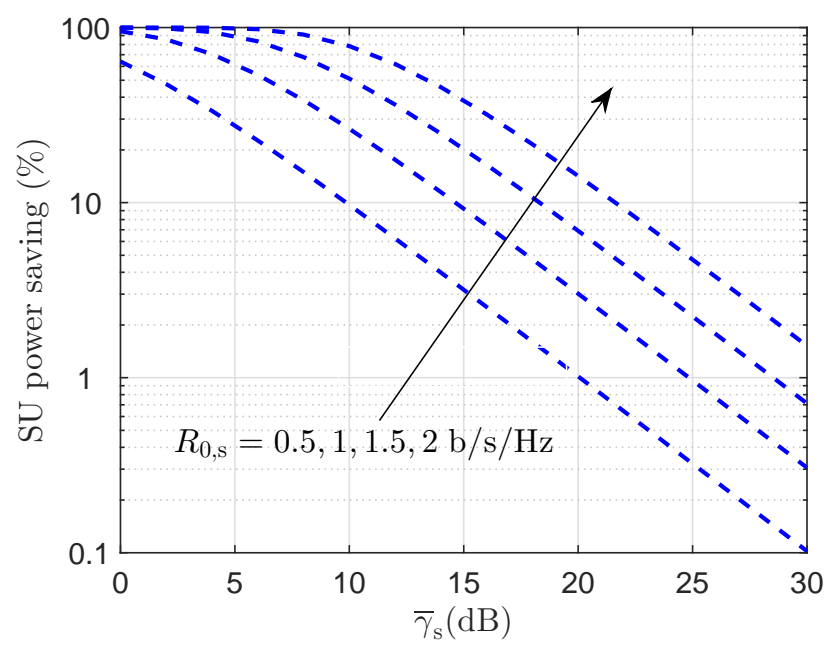

Fig. 7. Percentage of SU power saving of IDL-CSI improper based design versus $\bar{\gamma}_{\mathrm{s}}$ for $R_{0, \mathrm{~s}}=0.5,1,1.5,2 \mathrm{~b} / \mathrm{s} / \mathrm{Hz}$.

derived analytical expression (60). We assume $\kappa=5$ and $p_{\mathrm{c}}=1 \mathrm{~W}$. First, we observe that the simulation curve has a perfect match with the analytical expression. At lower SU target rates, the requirements for the $\mathrm{SU}$ are flexible and hence, both designs use less power and thus the average EE improves with increasing $\bar{\gamma}_{\mathrm{s}}$. On the other hand, as the SU rate increases, the requirements become more stringent, which force the SU to increase its transmit power and therefore, deteriorating the average EE performance. As $R_{0, \mathrm{~s}}$ increases,

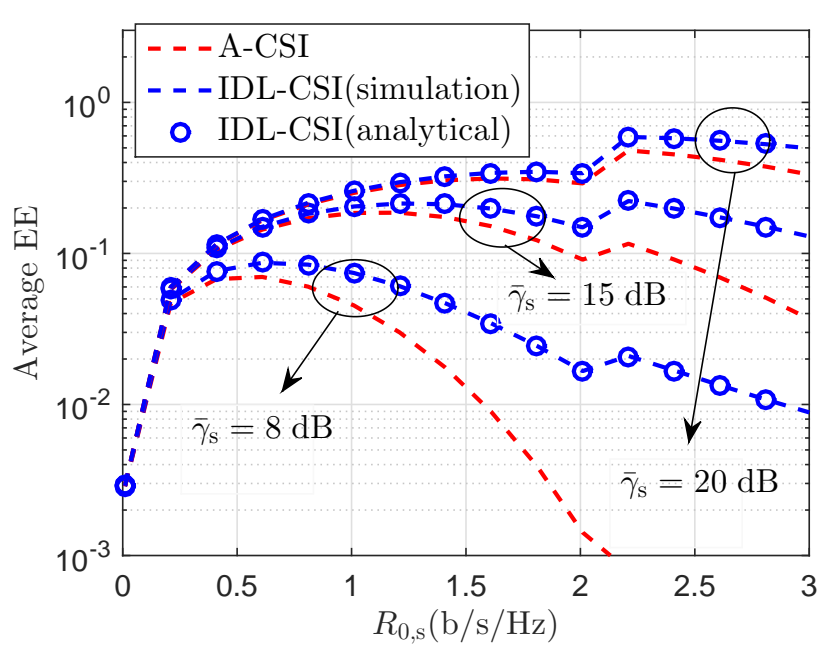

Fig. 8. SU average energy efficiency for A-CSI and IDL-CSI improper based designs versus $R_{0, \mathrm{~s}}$ for $\bar{\gamma}_{\mathrm{s}}=8,15,20 \mathrm{~dB}$.

the IDL-CSI based design uses less power to achieve the same target rate. The gap between the two designs increases as $\bar{\gamma}_{\mathrm{s}}$ decreases. Hence, the IDL-CSI based design can make use of the perfect knowledge of the CSI in order to save the transmit power and therefore boost the average EE of the SU system. We observe also from Fig. 8 that as $R_{0, \mathrm{~s}}$ increases, it reaches specific values at which the average EE behavior shows abrupt improvement. As we know from the improper condition (37) that there is a specific value of $R_{0, \mathrm{~s}}$, at which improper signaling can not be used, thus the solution switches to proper signaling at larger values $R_{0, \mathrm{~s}}$. Since the proper signaling scheme uses less power, it achieves better average EE which interprets this sudden improvement of the average EE performance.

Example 8: This example illustrates the benefits that can be reaped at the PUs side by the design based on perfect knowledge of $\gamma_{\mathrm{s}}$. For this purpose, we plot the PUs outage probability A-CSI and IDL-CSI based designs versus $\bar{\gamma}_{\mathrm{s}}$ for different $R_{0, \mathrm{~s}}$ in Fig. 9. The A-CSI based design PUs outage probability is fixed and does not change with $\bar{\gamma}_{\mathrm{s}}$ or $R_{0, \mathrm{~s}}$ as expected. On the other hand, the IDL-CSI based design has different performance. Specifically, at low $\bar{\gamma}_{\mathrm{s}}, P_{\text {saving }}$ has more value than higher values of $\bar{\gamma}_{\mathrm{s}}$ as can be shown in Fig. 7. Thus, PUs outage probability is expected to get more benefit as $\bar{\gamma}_{\mathrm{s}}$ decreases. Furthermore, As the SU target rate increases, the requirements for the $\mathrm{SU}$ become rigid and and the chances for the SU to stay idle become higher, which improves the PUs outage probability. 


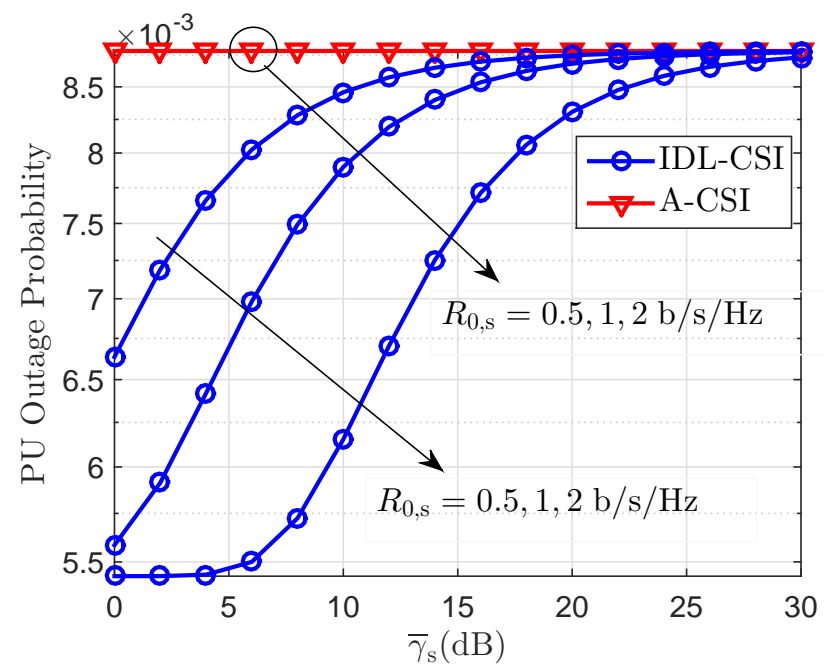

Fig. 9. PUs outage probability for A-CSI and IDL-CSI based designs versus $\bar{\gamma}_{\mathrm{s}}$ for $R_{0, \mathrm{~s}}=0.5,1,2 \mathrm{~b} / \mathrm{s} / \mathrm{Hz}$.

\section{CONCLUSION}

In this paper, we investigate the opportunity of sharing the spectrum resources of licensed FD PUs in underlay CR mode using improper Gaussian signaling. We use the outage probability as a performance metric, then derive a closed form for the SU and a tight upper bound for the PU. Based on the average CSI, we optimize the SU signal parameters, i.e., transmit power and circularity coefficient, to minimize the SU outage probability while maintaining a predetermined QoS requirements for the PU. As a result, we derive a low complexity algorithm that tunes the signal parameters to accomplish the design objectives. Moreover, we show that improper Gaussian signaling is beneficial if the maximum allowable INR at the PUs exceeds a predefined threshold and the SU works under a maximum allowable target rate. Then, we study the benefits of perfect knowledge of the SU direct link. Specifically, the SU can save more power and improve its average EE, and the PUs can achieve more protection in terms of its outage probability performance. The later merit may be used by other cognitive users in the same system to access the spectrum without deteriorating the PUs QoS. The numerical results show a promising performance for the improper Gaussian signaling with A-CSI and IDL-CSI. Specifically, the main advantage of the proposed scheme is for strong SU interference channels to PU, where proper signaling scheme tends to use less power, while SU with improper Gaussian signaling uses more power and compensates its interference impact through the increase of signal impropriety.

\section{APPENDiX A}

In this appendix, we prove that $p_{\mathrm{s}}^{(i)}\left(\mathcal{C}_{x}\right)$ in (31) is strictly increasing in $\mathcal{C}_{x}$ over the interval of interest $0<\mathcal{C}_{x}<1$. We assume here, that the $\mathrm{SU}$ is allowed to transmit, i.e., $\Upsilon_{i}>0$. The first derivative of $p_{\mathrm{s}}^{(i)}\left(\mathcal{C}_{x}\right)$ can be written as

$$
\frac{d p_{\mathrm{s}}^{(i)}\left(\mathcal{C}_{x}\right)}{d \mathcal{C}_{x}}=\frac{\mathcal{C}_{x}\left(\Phi_{i}-2 \Lambda_{i}\left(-\Lambda_{i}+\sqrt{\Lambda_{i}^{2}+\Phi_{i}}\right)\right)}{\Gamma_{\mathrm{p}_{i}} \overline{\mathcal{I}}_{\mathrm{s}_{j}}\left(1-\mathcal{C}_{x}^{2}\right)^{2} \sqrt{\Lambda_{i}^{2}+\Phi_{i}}},
$$

where $\Phi_{i}=\Gamma_{\mathrm{p}_{i}}\left(1-\mathcal{C}_{x}^{2}\right) \Upsilon_{i}$. Assuming that $R_{0, \mathrm{p}_{\mathrm{i}}}>0$, hence $\Phi_{i}>0$. From (63), if $\Lambda_{i} \leq 0$, it is always positive. On the other hand, if $\Lambda_{i}>0$ we can rewrite (63) as

$$
\frac{d p_{\mathrm{s}}^{(i)}\left(\mathcal{C}_{x}\right)}{d \mathcal{C}_{x}}=\frac{\mathcal{C}_{x} \Lambda_{i}\left(\left(\Omega_{i}+2\right)-\sqrt{\left(\Omega_{i}+2\right)^{2}-\Omega_{i}^{2}}\right)}{\Gamma_{\mathrm{p}_{i}} \overline{\mathcal{I}}_{\mathrm{s}_{j}}\left(1-\mathcal{C}_{x}^{2}\right)^{2} \sqrt{1+\Omega_{i}}},
$$

where $\Omega_{i}=\Phi_{i} / \Lambda_{i}^{2}$. It is clear that (64) is always positive.

\section{APPENDIX B}

In this appendix, we derive the conditions in (37) over $0<\mathcal{C}_{x}<1$ by using the chain rule of partial derivatives for $P_{\text {out }, \mathrm{s}}\left(\mathcal{G}_{m}, \mathcal{Y}\right), j \neq m$, in (36) with respect to $\mathcal{C}_{x}$,

$$
\frac{\partial P_{\text {out }, \mathrm{s}}\left(\mathcal{G}_{m}, \mathcal{Y}\right)}{\partial \mathcal{C}_{x}}=\frac{\partial P_{\text {out }, \mathrm{s}}}{\partial \mathcal{G}_{m}} \frac{d \mathcal{G}_{m}}{d \mathcal{C}_{x}}+\frac{\partial P_{\text {out }, \mathrm{s}}}{\partial \mathcal{Y}} \frac{d \mathcal{Y}}{d \mathcal{C}_{x}},
$$

which is simplified to be

$$
\begin{aligned}
& \frac{\partial P_{\mathrm{out}, \mathrm{s}}\left(\mathcal{G}_{m}, \mathcal{Y}\right)}{\partial \mathcal{C}_{x}}=(\underbrace{\frac{T \mathcal{C}_{x} \bar{\gamma}_{\mathrm{s}}\left(-\Lambda_{m}+\sqrt{\Lambda_{m}^{2}+\Phi_{m}}\right)}{\underbrace{}_{p_{j}} \overline{\mathcal{I}}_{\mathrm{s}_{j}}\left(\sqrt{1+\left(1-\mathcal{C}_{x}^{2}\right) \Gamma_{\mathrm{s}}}-1\right)\left(1-\mathcal{C}_{x}^{2}\right)}}_{\geq 0}) \times \\
& (\underbrace{\frac{\Lambda_{m}}{\sqrt{\Lambda_{m}^{2}+\Phi_{m}}}-\frac{1}{\sqrt{1+\left(1-\mathcal{C}_{x}^{2}\right) \Gamma_{\mathrm{s}}}}}_{\vartheta})
\end{aligned}
$$

where

$T=\frac{\left(\mathcal{Y} \mathcal{G}_{m}\left(\bar{\gamma}_{\mathrm{s}}\right)\left(\prod_{j=1}^{2} p_{j} \overline{\mathcal{I}}_{\mathrm{p}_{j}}+\Theta-\mathcal{Y}^{2} \mathcal{G}_{m}^{2}\left(\bar{\gamma}_{\mathrm{s}}\right)\right)+\Theta\right) \exp \left(-\frac{1}{\mathcal{Y} \mathcal{G}_{m}\left(\bar{\gamma}_{\mathrm{s}}\right)}\right)}{\Theta^{2}}$

and $\Theta=\prod_{j=1}^{2}\left(p_{j} \overline{\mathcal{I}}_{\mathrm{p}_{j}}+\mathcal{Y} \mathcal{G}_{m}\left(\bar{\gamma}_{\mathrm{s}}\right)\right)$. Here, we have two cases for $\Lambda_{m}$. If $\Lambda_{m} \leq 0$, then $\vartheta \leq 0$ and (66) is non-positive, hence, $P_{\text {out, }}\left(\mathcal{C}_{x}\right)$ is monotonically decreasing in $\mathcal{C}_{x}$. On the other hand, if $\Lambda_{m}>0$, then one can deduce easily that when $\Lambda_{m} \leq \sqrt{\Gamma_{\mathrm{p}_{m}} \Upsilon_{m} / \Gamma_{\mathrm{s}}}, \vartheta \leq 0$ and hence, $P_{\text {out }, \mathrm{s}}\left(\mathcal{C}_{x}\right)$ is monotonically decreasing in $\mathcal{C}_{x}$. Otherwise, if $\Lambda_{m}>\sqrt{\Gamma_{\mathrm{p}_{m}} \Upsilon_{m} / \Gamma_{\mathrm{s}}}$, $P_{\text {out }, \mathrm{s}}\left(\mathcal{C}_{x}\right)$ is monotonically increasing in $\mathcal{C}_{x}$. It is clear that the condition $\Lambda_{m} \leq \sqrt{\Gamma_{\mathrm{p}_{m}} \Upsilon_{m} / \Gamma_{\mathrm{s}}}$ combines both cases of $\Lambda_{m}$ for $P_{\text {out }, \mathrm{s}}\left(\mathcal{C}_{x}\right)$ to be monotonically decreasing in $\mathcal{C}_{x}$. Furthermore, this condition can be rewritten as

$$
R_{0, \mathrm{~s}} \leq \frac{1}{2} \log _{2}\left(1+\frac{\Gamma_{\mathrm{p}_{\mathrm{m}}} \Upsilon_{m}}{\Lambda_{m}^{2}}\right),
$$

which can be further simplified by inserting the expressions for $\Upsilon_{m}, \Lambda_{m}$ giving the condition (37).

\section{APPENDIX C}

In this appendix, we prove the monotonicity conditions of $P_{\text {out }, \mathrm{s}}^{\mathrm{DL}}\left(\mathcal{G}_{m}, \mathcal{Y}\right), j \neq m$, in (51) over $0<\mathcal{C}_{x}<1$. We assume here that the transmission condition in (45) is satisfied, i.e., the $\mathrm{SU}$ is allowed to transmit. By using the chain rule of partial derivatives as in Appendix B, we get

$$
\begin{aligned}
\frac{\partial P_{\mathrm{out}, \mathrm{s}}^{\mathrm{DL}-\mathrm{T}}\left(\mathcal{G}_{m}, \mathcal{Y}\right)}{\partial \mathcal{C}_{x}}= & M \times \underbrace{\frac{\mathcal{C}_{x} \gamma_{\mathrm{s}}\left(-\Lambda_{m}+\sqrt{\Lambda_{m}^{2}+\Phi_{m}}\right)}{\Gamma_{p_{m}} \overline{\mathcal{I}}_{\mathrm{s}_{j}}\left(\sqrt{1+\left(1-\mathcal{C}_{x}^{2}\right) \Gamma_{\mathrm{s}}}-1\right)\left(1-\mathcal{C}_{x}^{2}\right)}}_{\geq 0} \times \\
& \left(\frac{\Lambda_{m}}{\sqrt{\Lambda_{m}^{2}+\Phi_{m}}}-\frac{1}{\sqrt{1+\left(1-\mathcal{C}_{x}^{2}\right) \Gamma_{\mathrm{s}}}}\right)
\end{aligned}
$$


where

$$
M=\left\{\begin{array}{cl}
\sum_{\substack{i=1 \\
j \neq i}}^{2} \frac{\mathcal{K}_{i, j}}{p_{i} \mathcal{I}_{\mathrm{p}_{i}}} \exp \left(-\frac{\mathcal{Y G} \mathcal{G}_{m}\left(\gamma_{\mathrm{s}}\right)-1}{p_{i} \mathcal{I}_{\mathrm{p}_{i}}}\right), & \text { if } \mathrm{p}_{1} \overline{\mathcal{I}}_{\mathrm{p}_{1}} \neq \mathrm{p}_{2} \overline{\mathcal{I}}_{\mathrm{p}_{2}} \\
\left(\frac{\mathcal{Y G} \mathcal{G}_{m}\left(\gamma_{\mathrm{s}}\right)-1}{\left(p_{1} \overline{\mathcal{I}}_{\mathrm{p}_{1}}\right)^{2}}\right) \exp \left(-\frac{\mathcal{Y G} \mathcal{G}_{m}\left(\gamma_{\mathrm{s}}\right)-1}{p_{1} \mathcal{I}_{\mathrm{p}_{1}}}\right), & \text { if } \mathrm{p}_{1} \overline{\mathcal{I}}_{\mathrm{p}_{1}}=\mathrm{p}_{2} \overline{\mathcal{I}}_{\mathrm{p}_{2}}
\end{array}\right.
$$

For instance, if $M$ is non-negative and when the condition in (37) is valid, then, $\vartheta \leq 0$. As a result, (69) is non-positive and $P_{\text {out }, \mathrm{s}}^{\mathrm{DL}-\mathrm{T}}$ is monotonically decreasing in $\mathcal{C}_{x}$. On the other hand, if the condition in (37) does not hold, then $\vartheta>0$ and therefore, $P_{\text {out }, \mathrm{s}}^{\mathrm{DL}} \mathrm{T}$ is monotonically increasing in $\mathcal{C}_{x}$. To this point, we need now to show that $M$ is non-negative. For asymmetric PUs interference links, (70) can be expressed as

$$
\begin{aligned}
M= & \frac{1}{p_{1} \overline{\mathcal{I}}_{\mathrm{p}_{1}}-p_{2} \overline{\mathcal{I}}_{\mathrm{p}_{2}}} \times \\
& \left(\exp \left(-\frac{\mathcal{Y} \mathcal{G}_{m}\left(\gamma_{\mathrm{s}}\right)-1}{p_{1} \overline{\mathcal{I}}_{\mathrm{p}_{1}}}\right)-\exp \left(-\frac{\mathcal{Y} \mathcal{G}_{m}\left(\gamma_{\mathrm{s}}\right)-1}{p_{2} \overline{\mathcal{I}}_{\mathrm{p}_{2}}}\right)\right) .
\end{aligned}
$$

Here, we have two cases, either $p_{1} \overline{\mathcal{I}}_{\mathrm{p}_{1}}>p_{2} \overline{\mathcal{I}}_{\mathrm{p}_{2}}$ or $p_{1} \overline{\mathcal{I}}_{\mathrm{p}_{1}}<$ $p_{2} \overline{\mathcal{I}}_{\mathrm{p}_{2}}$. For the first, it is clear that $\mathrm{M} \geq 0$. For the second, the same argument applies by replacing every index 1 by 2 and vice versa in (71). For the other scenario, when $p_{1} \overline{\mathcal{I}}_{\mathrm{p}_{1}}=p_{2} \overline{\mathcal{I}}_{\mathrm{p}_{2}}$, we can write $M$ as

$$
M=\frac{\zeta\left(p_{\mathrm{s}}^{(m)}, \mathcal{C}_{x}\right)}{\left(p_{1} \overline{\mathcal{I}}_{\mathrm{p}_{1}}\right)^{2}} \exp \left(-\frac{\zeta\left(p_{\mathrm{s}}^{(m)}, \mathcal{C}_{x}\right)}{\left(p_{1} \overline{\mathcal{I}}_{\mathrm{p}_{1}}\right)^{2}}\right),
$$

which is non-negative by following (45).

\section{APPENDIX D}

In this appendix, we aim at deriving the equivalent optimization problem in (53). We assume here that the transmission condition in (45) is fulfilled, i.e., the $\mathrm{SU}$ is allowed to transmit. First, we can write $P_{\text {out }, \mathrm{s}}^{\mathrm{DL}}\left(p_{o}^{(z)}, \mathcal{C}_{o}^{(z)}\right)$ in (52), when $p_{1} \overline{\mathcal{I}}_{\mathrm{p}_{1}} \neq p_{2} \overline{\mathcal{I}}_{\mathrm{p}_{2}}$, as

$$
\begin{aligned}
& P_{\mathrm{out}, \mathrm{s}}^{\mathrm{DL}-\mathrm{T}}\left(p_{o}^{(z)}, \mathcal{C}_{o}^{(z)}\right)=\frac{1}{p_{1} \overline{\mathcal{I}}_{\mathrm{p}_{1}}-p_{2} \overline{\mathcal{I}}_{\mathrm{p}_{2}}} \times \\
& \left(p_{1} \overline{\mathcal{I}}_{\mathrm{p}_{1}} \exp \left(-\frac{\gamma_{\mathrm{s}} \Delta-1}{p_{1} \overline{\mathcal{I}}_{\mathrm{p}_{1}}}\right)-p_{2} \overline{\mathcal{I}}_{\mathrm{p}_{2}} \exp \left(-\frac{\gamma_{\mathrm{s}} \Delta-1}{p_{2} \overline{\mathcal{I}}_{\mathrm{p}_{2}}}\right)\right),
\end{aligned}
$$

where $\Delta=\frac{\left(1-\left(\mathcal{C}_{o}^{(z)}\right)^{2}\right)}{\Psi_{\mathrm{s}}\left(p_{o}^{(z)}, \mathcal{C}_{o}^{(z)}\right)}$. Similar to the proof in Appendix C, we assume, first, that $p_{1} \overline{\mathcal{I}}_{\mathrm{p}_{1}}>p_{2} \overline{\mathcal{I}}_{\mathrm{p}_{2}}$. We calculate the first derivative of (73) with respect to $\Delta$ as

$$
\begin{aligned}
\frac{d P_{\mathrm{out}, \mathrm{s}}^{\mathrm{DL}-\mathrm{T}}}{d \Delta}= & \frac{\gamma_{\mathrm{s}}}{p_{1} \overline{\mathcal{I}}_{\mathrm{p}_{1}}-p_{2} \overline{\mathcal{I}}_{\mathrm{p}_{2}}} \times \\
& \left(\exp \left(-\frac{\gamma_{\mathrm{s}} \Delta-1}{p_{2} \overline{\mathcal{I}}_{\mathrm{p}_{2}}}\right)-\exp \left(-\frac{\gamma_{\mathrm{s}} \Delta-1}{p_{1} \overline{\mathcal{I}}_{\mathrm{p}_{1}}}\right)\right)
\end{aligned}
$$

which is always non-positive, independent of the value of $\gamma_{\mathrm{s}}$, and hence, $P_{\text {out }, \mathrm{s}}^{\mathrm{DL}-\mathrm{T}}\left(p_{o}^{(z)}, \mathcal{C}_{o}^{(z)}\right)$ is monotonically decreasing in $\Delta$. Thus, we can replace the original optimization problem by maximizing $\Delta$ over the optimal local solution pairs $\left(p_{o}^{(z)}, \mathcal{C}_{o}^{(z)}\right)$ to obtain the global pair $\left(p_{\mathrm{s}}^{*}, \mathcal{C}_{x}^{*}\right)$ which yields (53). Also, For the other case in which $p_{1} \overline{\mathcal{I}}_{\mathrm{p}_{1}}<p_{2} \overline{\mathcal{I}}_{\mathrm{p}_{2}}$, the same argument applies as illustrated in Appendix C. On the other hand, when $p_{1} \overline{\mathcal{I}}_{\mathrm{p}_{1}}=p_{2} \overline{\mathcal{I}}_{\mathrm{p}_{2}}$, we can write the derivative as

$$
\frac{d P_{\mathrm{out}, \mathrm{s}}^{\mathrm{DL}-\mathrm{T}}}{d \Delta}=-\frac{\gamma_{\mathrm{s}}}{\bar{p}_{1}}\left(\frac{\gamma_{\mathrm{s}} \Delta-1}{\overline{\mathrm{I}}_{1}}\right) \exp \left(-\frac{\gamma_{\mathrm{s}} \Delta-1}{p_{1} \overline{\mathcal{I}}_{\mathrm{p}_{1}}}\right)
$$

which, from the transmission condition in (45), it follows that the derivative is non-positive. Hence, same illustrated arguments apply and this concludes the proof.

\section{REFERENCES}

[1] J. Mitola III and G. Q. Maguire Jr, "Cognitive radio: making software radios more personal," IEEE Personal Commun. Mag., vol. 6, no. 4, pp. 13-18, Aug. 1999.

[2] Q. Zhao and B. M. Sadler, "A survey of dynamic spectrum access," IEEE Signal Process. Mag., vol. 24, no. 3, pp. 79-89, May 2007.

[3] D. Kim, H. Lee, and D. Hong, "A survey of in-band full-duplex transmission: From the perspective of PHY and MAC layers," IEEE Commun. Surveys Tuts., vol. 17, no. 4, pp. 2017-2046, 2015.

[4] A. Sabharwal, P. Schniter, D. Guo, D. W. Bliss, S. Rangarajan, and R. Wichman, "In-band full-duplex wireless: Challenges and opportunities," IEEE J. Sel. Areas Commun., vol. 32, no. 9, pp. 1637-1652, Sep. 2014.

[5] Z. Zhang, X. Chai, K. Long, A. V. Vasilakos, and L. Hanzo, "Full duplex techniques for $5 \mathrm{G}$ networks: self-interference cancellation, protocol design, and relay selection," IEEE Commun. Mag., vol. 53, no. 5, pp. 128-137, May 2015.

[6] H. Kim, S. Lim, H. Wang, and D. Hong, "Optimal power allocation and outage analysis for cognitive full duplex relay systems," IEEE Trans. Wireless Commun., vol. 11, no. 10, pp. 3754-3765, Oct. 2012.

[7] B. Zhong, Z. Zhang, X. Chai, Z. Pan, K. Long, and H. Cao, "Performance analysis for opportunistic full-duplex relay selection in underlay cognitive networks," IEEE Trans. Veh. Technol. DOI: 10.1109/TVT.2014.2368584.

[8] W. Afifi and M. Krunz, "Incorporating self-interference suppression for full-duplex operation in opportunistic spectrum access systems," IEEE Trans. Wireless Commun., vol. 14, no. 4, pp. 2180-2191, April 2015.

[9] V. R. Cadambe, S. A. Jafar, and C. Wang, "Interference alignment with asymmetric complex signaling: settling the høst-madsen-nosratinia conjecture," IEEE Trans. Inf. Theory, vol. 56, no. 9, pp. 4552-4565, Sep. 2010.

[10] Z. Ho and E. Jorswieck, "Improper Gaussian signaling on the two-user SISO interference channel," IEEE Trans. Wireless Commun., vol. 11, no. 9, pp. 3194-3203, Sep. 2012.

[11] Y. Zeng, C. M. Yetis, E. Gunawan, Y. L. Guan, and R. Zhang, "Transmit optimization with improper Gaussian signaling for interference channels," IEEE Trans. Signal Process., vol. 61, no. 11, pp. 2899-2913, Jun. 2013.

[12] Y. Zeng, R. Zhang, E. Gunawan, and Y. Guan, "Optimized transmission with improper Gaussian signaling in the K-user MISO interference channel," IEEE Trans. Wireless Commun., vol. 12, no. 12, pp. 63036313, Dec. 2013.

[13] E. Kurniawan and S. Sun, "Improper gaussian signaling scheme for the Z-interference channel," IEEE Trans. Wireless Commun., vol. 14, no. 7, pp. 3912-3923, Jul. 2010.

[14] M. Gaafar, M. G. Khafagy, O. Amin, and M. S. Alouini, "Improper gaussian signaling in full-duplex relay channels with residual selfinterference," in Proc. IEEE Int. Conf. on Commun. (ICC), May 2016, pp. 1-7.

[15] M. Gaafar, O. Amin, A. Ikhlef, A. Chaaban, and M. S. Alouini, "On alternate relaying with improper gaussian signaling," IEEE Commun. Lett., vol. 20, no. 8, pp. 1683-1686, Aug. 2016.

[16] C. Lameiro, I. Santamaria, and P. Schreier, "Benefits of improper signaling for underlay cognitive radio," IEE Wireless Commun. Lett., vol. 4, no. 1, pp. 22-25, Feb. 2015.

[17] _ "Analysis of maximally improper signaling schemes for underlay cognitive radio networks," in Proc. IEEE Int. Conf. Commun. (ICC), London, UK., 2015, pp. 1398-1403. 
[18] O. Amin, W. Abediseid, and M.-S. Alouini, "Outage performance of cognitive radio systems with improper Gaussian signaling," in Proc. IEEE International Symposium on Information Theory (ISIT), Hong Kong, 2015, pp. 1851-1855.

[19] M. Gaafar, O. Amin, W. Abediseid, and M.-S. Alouini, "Spectrum sharing opportunities of Full-Duplex systems using improper gaussian signaling," in Proc. Personal, Indoor and Mobile Radio Commun. (PIMRC), Hong Kong, China, Aug. 2015, pp. 244-249.

[20] — "Sharing the licensed spectrum of full-duplex systems using improper Gaussian signaling," in Proc. IEEE Global Commun. Conf. (GLOBCOM), San Diego, USA, Dec. 2015, pp. 1-6.

[21] F. D. Neeser and J. L. Massey, "Proper complex random processes with applications to information theory," IEEE Trans. Inf. Theory, vol. 39, no. 4, pp. 1293-1302, Jul. 1993.

[22] T. Kwon, S. Lim, S. Choi, and D. Hong, "Optimal duplex mode for DF relay in terms of the outage probability," IEEE Trans. Veh. Technol. vol. 59, no. 7, pp. 3628-3634, Sep. 2010.

[23] T. P. Do and T. V. T. Le, "Power allocation and performance comparison of full duplex dual hop relaying protocols," IEEE Commun. Lett., vol. 19, no. 5, pp. 791-794, May 2015.

[24] M. G. Khafagy, A. Ismail, M.-S. Alouini, and S. Aïssa, "Efficient cooperative protocols for full-duplex relaying over nakagami-fading channels," IEEE Trans. Wireless Commun., vol. 14, no. 6, pp. 34563470, Jun. 2015

[25] M. Mohammadi, B. K. Chalise, H. A. Suraweera, C. Zhong, G. Zheng, and I. Krikidis, "Throughput analysis and optimization of wirelesspowered multiple antenna full-duplex relay systems," IEEE Trans. Commun., vol. 64, no. 4, pp. 1769-1785, Apr. 2016.

[26] E. Biglieri, R. Calderbank, A. Constantinides, A. Goldsmith, A. Paulraj, and $\mathrm{H}$. V. Poor, MIMO wireless communications. Cambridge University Press, 2007.

[27] S. P. Boyd and L. Vandenberghe, Convex Optimization. Cambridge University Press, 2004.

[28] I. Gradstein and I. Ryszik, Tables of integrals, sums, and products. Academic Press New York, 1980

[29] H. Holma and A. Toskala, WCDMA for umts: hspa evolution and lte. John Wiley \& Sons, 2010.

[30] S. M. Ross, Introduction to probability models. Academic press, 2010.

[31] C. Forbes, M. Evans, N. Hastings, and B. Peacock, Statistical distributions. John Wiley \& Sons, 2011.

[32] O. Amin, E. Bedeer, M. H. Ahmed, O. Dobre et al., "A novel energy efficient scheme with a finite-rate feedback channel," IEEE Wireless Commun. Lett., vol. 3, no. 5, pp. 497-500, Oct. 2014.

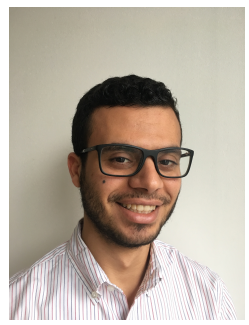

Mohamed Gaafar (S'15) was born in Bani Souwaif, Egypt. He received the B. Sc. degree (with highest honors) in Communications Engineering from the German University in Cairo (GUC), Egypt in 2013 (Last semester of the B. Sc. studies was done at Ulm University, Germnay). He received the M. Sc. degree in Electrical Engineering from King Abdullah University of Science and Technology (KAUST), Saudi Arabia in $2016 . \mathrm{He}$ is currently working towards his $\mathrm{Ph} . \mathrm{D}$. in Electrical Engineering and Computer Science at the Technical University of Berlin (TU Berlin), Germany. His current research interests include information theory, wireless communications and machine learning.

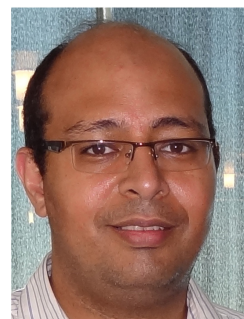

Osama Amin (S'07, M'11, SM'15) received B. Sc. degree in Electrical and Electronics Engineering from Aswan University, Aswan, Egypt, in 2000, M. Sc. degree in Electrical Engineering from Assiut University, Assiut, Egypt in 2004 and Ph.D. degree in Electrical and Computer Engineering, University of Waterloo, Canada in 2010. In June 2012, he joined Assiut University as an Assistant Professor in the Electrical and Electronics Engineering department. Currently, he is with King Abdullah University of Science and Technology (KAUST), Thuwal, Makkah, Kingdom of Saudi Arabia. His general research interests lie in communications systems and signal processing for communications with special emphasis on wireless applications. Specific research areas include green communications, cognitive radio, cooperative communications, and channel estimation.

Dr. Amin is an Editor for the IEEE Communications Letters. He has served as a technical program committee (TPC) member for ICC, IEEE VTC, CROWNCOM, PIMRC, and ISSPIT conferences. He has served also as co-organizer and a co-chair of the Next Generation Green ICT and 5G Networking (GreeNets) 2015 in the IEEE International Conference on Ubiquitous Wireless Broadband (ICUWB) in Montreal, Canada.

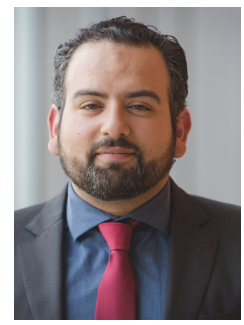

Walid Abediseid (S'04, M'11) was born in Etobicoke, Ontario, Canada. He received the B. Sc. and M. Sc. degrees in Electrical Engineering from the University of Ottawa, Canada, in 2004 and 2007, respectively. He then received his Ph.D. from the Department of Electrical and Computer Engineering, University of Waterloo in 2011. He was a postdoctoral fellow at King Abdullah University of Science and Technology (KAUST), Thuwal, Makkah Province, Saudi Arabia, from December 2011 to December 2013. His research interests included coding and information theory, MIMO wireless communication systems, lattice applications for digital and wireless communications, detection and estimation.

In January 2014, Dr. Abediseid joined the Saudi Initiatives department at KAUST where he holds the academic development specialist of the Saudi Research Science Institute. He also serves as a tutor during the SRSI summer program that provides support to students conducting research in STEM fields.

Dr. Abediseid was a recipient of many prestigious awards during his graduate studies such as the Research In Motion Graduate Scholarship from 2008 to 2009, and the NSERC Alexander Graham Bell Canada Graduate Scholarshipone of Canada's most prestigious graduate awards from 2009 to 2011.

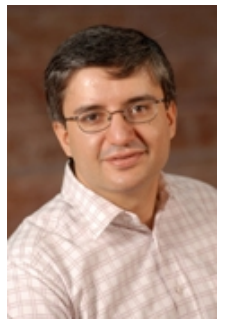

Mohamed-Slim Alouini (S'94, M'98, SM'03, F'09) was born in Tunis, Tunisia. He received the Ph.D. degree in Electrical Engineering from the California Institute of Technology (Caltech), Pasadena, CA, USA, in 1998. He served as a faculty member in the University of Minnesota, Minneapolis, MN, USA, then in the Texas A\&M University at Qatar, Education City, Doha, Qatar before joining King Abdullah University of Science and Technology (KAUST), Thuwal, Makkah Province, Saudi Arabia as a Professor of Electrical Engineering in 2009. His current research interests include the modeling, design, and performance analysis of wireless communication systems. 